\title{
Beautiful mathematics for beauty-full and other multi-heavy hadronic systems
}

\author{
K. Azizi ${ }^{1,2}{ }^{*}$, A. R. Olamaei ${ }^{3} \dagger$, S. Rostami ${ }^{4}$ \\ ${ }^{1}$ Department of Physics, Doğuş University, Acibadem-Kadiköy, 34722 Istanbul, Turkey \\ ${ }^{2}$ School of Physics, Institute for Research in Fundamental Sciences (IPM) \\ P. O. Box 19395-5531, Tehran, Iran \\ ${ }^{3}$ Department of Physics, Jahrom University, Jahrom, P. O. Box 74137-66171, Iran \\ ${ }^{4}$ Young Researchers and Elites Club, South Tehran Branch, Islamic Azad University, Tehran, Iran
}

\begin{abstract}
In most non-perturbative methods in hadron physics the calculations are started with a correlation function in terms of some interpolating and transition currents in $x$-space. For simplicity, the calculations are then transformed to the momentum space by a Fourier transformation. To suppress the contributions of the higher states and continuum; and enhance the ground state contribution, Borel transformation as well as continuum subtraction are applied by the help of quark-hadron duality assumption. In the present study we work out the mathematics required for these processes in the case of light and multiheavy hadrons. We address a well-known problem in subtraction of the effects of the higher states and continuum and discuss how we find finite results without any divergence by using an appropriate representation of the modified Bessel functions, appearing in the heavy quark propagator, and successive applications of the Borel transformations, which lead to more suppression of the higher states and continuum contributions. The results obtained can be used in determination of the spectroscopic and decay properties of the multi-heavy standard and non-conventional (exotic) systems in many non-perturbative methods, specially the QCD sum rules.
\end{abstract}

\footnotetext{
*e-mail: kazizi@dogus.edu.tr

${ }^{\dagger}$ e-mail: olamaei@jahromu.ac.ir

${ }_{\ddagger}$ e-mail: Asalrostami.phy@gmail.com
} 


\section{Introduction}

The traditional mesons and baryons as strong bound states of quarks and gluons are represented as the standard $q \bar{q}$ and $q q q$ states in terms of the valence quarks. There have been made good progresses on identification and determination of many spectroscopic and decay properties of theses standard hadrons both experimentally and theoretically (for more information see for instance [1,4] and references therein). Indeed, many ground and higher states at different light and heavy channels have been discovered in the experiment. Roughly, all the light and heavy ground states mesons predicted by the quark model, have been observed. Besides, all the light and single charmed ground state baryons together with some excited states have been detected by different experiments, as well. In the case of heavy $b$-baryons, except the $\Omega_{b}^{*}$ baryon with spin- $\frac{3}{2}$, all single heavy baryons have been seen. For the standard baryons with two or three heavy quarks, however, only the $\Xi_{c c}$ has been discovered. For the first time, its existence was reported by SELEX collaboration [5, 6], but it was not been later confirmed by other groups. Recently, the LHCb collaboration has reported the observation of doubly heavy baryon $\Xi_{c c}^{++}$via the decay mode $\Lambda_{c}^{+} K^{-} \pi^{+} \pi$ with mass $3621.40 \pm 0.72$ (stat.) \pm 0.27 (syst.) $\pm 0.14\left(\Lambda_{c}^{+}\right) \mathrm{MeV} / c^{2}$ [7].

Neither the quark model, nor the QCD as the theory of the strong interaction do not exclude the existence of the strong bound states out of the traditional $q \bar{q}$ and $q q q$ systems $[8] 12$. The existence of such non-conventional (exotic) states were predicted by Jaffe [8,13]. Although they had been predicted more than forty years ago, the experimental searches had ended up in null results up to 2003, when the Belle Collaboration discovered the famous $X(3872)$ in the $\pi^{+} \pi^{-} j / \psi$ invariant mass distribution from $B^{ \pm} \rightarrow K^{ \pm} \pi^{+} \pi^{-} J / \psi$ decays [14]. This discovery had stimulated the experimental and theoretical investigations of the exotic states such that a plenty of tetraquarks were discovered by different experimental collaborations.

Motivated by these progresses, in 2015, the LHCb Collaboration announced the observation of two pentaquark states $P_{c}^{+}(4380)$ and $P_{c}^{+}(4450)$ in the $J / \psi p$ spectrum of $\Lambda_{b} \rightarrow$ $J / \psi p K^{-}$process [15]. After discovery of the exotic states, many theoretical and experimental efforts have been devoted to the determination of the internal structure of these new objects. Despite a lot of studies, unfortunately, the nature and structure of most of exotic states remain unclear. The studies predict a hidden charm/bottom structure together with two or three light quarks for the tetraquark or pentaquark states newly discovered by experiments. However, there are many contradictory suggestions on the quark organizations of these states in the literature (for more information see for example [16-18] and references therein). Hence, we need more study on the spectroscopic and decay properties of the newly founded exotic and heavy baryonic states.

Up to now, studies of hadrons with light and one heavy quark have well covered (see for instance Refs. [18 31] and references therein) but investigations of hadrons with two or more heavy flavour quarks are still rare (see for example Refs. [32,36, 38, 41] and references therein). In order to study the properties of heavy systems containing more heavy quarks using some non-perturbative methods, especially the QCD sum rules, we should develop the mathematics of heavy and light systems with more quarks. The situation in calculations of the spectroscopic parameters of such states and corresponding mathematics is better. Indeed, in [32-36], the mathematics required for performing the Fourier and Borel transformations as well as the continuum subtraction related to the spectroscopic parameters of the doubly and triply heavy baryons has been well worked out. We present the mathematics required to calculate all physical properties of the baryons with two-five heavy quarks, especially their electromagnetic and strong decays and their interactions with other particles. A special case of these baryons with full $b$ quarks and without light quarks are called beauty-full states (see for instance [37]). We use the phrase "beautiful mathematics" in the title of the manuscript to insist that the results are finite and the calculations do not include any divergence.

Similar calculations for the light and single heavy systems had been done for interaction 
with photon $\left(q^{2}=0\right)$ in Ref. [42] and in the case of single heavy baryons with $q^{2} \neq 0$ in Ref. [22. We extend these calculations to study the heavy systems containing two-five heavy quarks for all values of the transferred momentum squared. For reader we repeat the calculations done in Refs. 22, 42, for the light and heavy systems to give an idea about the calculations for simple systems.

This article is structured in the following way. Section 2 is devoted to developing the mathematics of the light and heavy systems, each of them is explained in details in separate sub-sections. In section 3 we present the concluding remarks.

\section{Mathematics for light and heavy systems}

As we previously mentioned, in many non-perturbative methods, especially the QCD sum rules (traditional SVZ and light cone QCD [43,44]), in order to calculate the physical parameters of hadrons as strong bound states of quarks and gluons, we start with a basic object called correlation function. This function is expressed in terms of time ordering product of some interpolating or transition currents in coordinate space. As an example let us consider the strong interaction of doubly heavy spin- $1 / 2$ baryons with light pseudoscalar mesons. The light-cone correlation function responsible for such vertices can be written as

$$
\Pi=i \int d^{4} x e^{i p x}\langle\mathcal{P}(q)|\mathcal{T}\{\eta(x) \bar{\eta}(0)\}| 0\rangle,
$$

where $\mathcal{P}(q)$ denotes the pseudoscalar mesons of momentum $q$. In light-cone QCD sum rules we use the distribution amplitudes (DAs) of these mesons expanded in terms of wave functions having different twists (for details about DAs of the pseudoscalar particles please see the Appendix). In Eq. (11) $\eta$ represents the interpolating currents of the doubly heavy baryons. It is given as

$$
\begin{aligned}
\eta^{S} & =\frac{1}{\sqrt{2}} \epsilon_{a b c}\left\{\left(Q^{a T} C q^{b}\right) \gamma_{5} Q^{\prime c}+\left(Q^{\prime a T} C q^{b}\right) \gamma_{5} Q^{c}+\beta\left(Q^{a T} C \gamma_{5} q^{b}\right) Q^{\prime c}+\beta\left(Q^{\prime a T} C \gamma_{5} q^{b}\right) Q^{c}\right\} \\
\eta^{A} & =\frac{1}{\sqrt{6}} \epsilon_{a b c}\left\{2\left(Q^{a T} C Q^{\prime b}\right) \gamma_{5} q^{c}+\left(Q^{a T} C q^{b}\right) \gamma_{5} Q^{\prime c}-\left(Q^{\prime a T} C q^{b}\right) \gamma_{5} Q^{c}+2 \beta\left(Q^{a T} C \gamma_{5} Q^{\prime b}\right) q^{c}\right. \\
& \left.+\beta\left(Q^{a T} C \gamma_{5} q^{b}\right) Q^{\prime c}-\beta\left(Q^{\prime a T} C \gamma_{5} q^{b}\right) Q^{c}\right\},
\end{aligned}
$$

where $S$ and $A$ respectively represent the symmetric and anti-symmetric parts, $\beta$ is a mixing parameter with $\beta=-1$ corresponding to the famous Ioffe current, $C$ is charge conjugation operator; and $a, b$, and $c$ are the color indices. Here $Q / Q^{\prime}$ and $q$ correspond to the heavy and light quarks fields, respectively. The interpolating current with the $u$ or $d$ quark corresponds to the $\Xi_{Q Q q}$, but with $s$ indicates the $\Omega_{Q Q q}$ baryons, respectively. Note that in the symmetric part, both heavy quarks can be identical or different, but in the anti-symmetric part two heavy quarks should be different. After insertion of the above currents into the correlation function and contracting out the quark-pairs using the Wick's theorem, we get a result in 
terms of the heavy and light quarks' propagators. For instance for the symmetric part we get

$$
\begin{aligned}
\Pi^{S} & =i \mathcal{A} \epsilon_{a b c} \epsilon_{a^{\prime} b^{\prime} c^{\prime}} \int d^{4} x e^{i p x}\langle\mathcal{P}(q)|\left\{-\gamma_{5} S_{Q}^{c b^{\prime}} S_{q}^{\prime b a^{\prime}} S_{Q^{\prime}}^{a c^{\prime}} \gamma_{5}-\gamma_{5} S_{Q^{\prime}}^{c b^{\prime}} S_{q}^{\prime b a^{\prime}} S_{Q}^{a c^{\prime}} \gamma_{5}\right. \\
& +\gamma_{5} S_{Q^{\prime}}^{c c^{\prime}} \gamma_{5} \operatorname{Tr}\left[S_{Q}^{a b^{\prime}} S_{q}^{\prime b a^{\prime}}\right]+\gamma_{5} S_{Q}^{c c^{\prime}} \gamma_{5} \operatorname{Tr}\left[S_{Q^{\prime}}^{a b^{\prime}} S_{q}^{\prime b a^{\prime}}\right] \\
& +\beta\left(-\gamma_{5} S_{Q}^{c b^{\prime}} \gamma_{5} S_{q}^{\prime b a^{\prime}} S_{Q^{\prime}}^{a c^{\prime}}-\gamma_{5} S_{Q^{\prime}}^{c b^{\prime}} \gamma_{5} S_{q}^{\prime b a^{\prime}} S_{Q}^{a c^{\prime}}-S_{Q}^{c b^{\prime}} S_{q}^{\prime b a^{\prime}} \gamma_{5} S_{Q^{\prime}}^{a c^{\prime}} \gamma_{5}\right. \\
& -S_{Q^{\prime}}^{c b^{\prime}} S_{q}^{\prime b a^{\prime}} \gamma_{5} S_{Q}^{a c^{\prime}} \gamma_{5}+\gamma_{5} S_{Q^{\prime}}^{c c^{\prime}} \operatorname{Tr}\left[S_{Q}^{a b^{\prime}} \gamma_{5} S_{q}^{\prime b a^{\prime}}\right]+S_{Q^{\prime}}^{c c c^{\prime}} \gamma_{5} \operatorname{Tr}\left[S_{Q}^{a b^{\prime}} S_{q}^{\prime b a^{\prime}} \gamma_{5}\right] \\
& \left.+\gamma_{5} S_{Q}^{c c^{\prime}} \operatorname{Tr}\left[S_{Q^{\prime}}^{a b^{\prime}} \gamma_{5} S_{q}^{\prime b a^{\prime}}\right]+S_{Q}^{c c^{\prime}} \gamma_{5} \operatorname{Tr}\left[S_{Q^{\prime}}^{a b^{\prime}} S_{q}^{\prime b a^{\prime}} \gamma_{5}\right]\right) \\
& +\beta^{2}\left(-S_{Q}^{c b^{\prime}} \gamma_{5} S_{q}^{\prime b a^{\prime}} \gamma_{5} S_{Q^{\prime}}^{a c^{\prime}}-S_{Q^{\prime}}^{c b^{\prime}} \gamma_{5} S_{q}^{\prime b a^{\prime}} \gamma_{5} S_{Q}^{a c^{\prime}}\right. \\
& \left.\left.+S_{Q^{\prime}}^{c c^{\prime}} \operatorname{Tr}\left[S_{q}^{b a^{\prime}} \gamma_{5} S_{Q}^{\prime a b^{\prime}} \gamma_{5}\right]+S_{Q}^{c c c^{\prime}} \operatorname{Tr}\left[S_{q}^{b a^{\prime}} \gamma_{5} S_{Q^{\prime}}^{\prime a b^{\prime}} \gamma_{5}\right]\right)\right\}|0\rangle
\end{aligned}
$$

where $S^{\prime}=C S^{T} C$, with $S$ being the heavy or light quark propagator, and $\mathcal{A}$ is normalization constant. To proceed, we need to know the explicit expressions of the light and heavy quark propagators in $x$-space. They are given as [18,45,47]

$$
\begin{aligned}
S_{q}(x)= & \frac{i \not x}{2 \pi^{2} x^{4}}-\frac{m_{q}}{4 \pi^{2} x^{2}}-\frac{\langle\bar{q} q\rangle}{12}\left(1-i \frac{m_{q}}{4} \not x\right)-\frac{x^{2}}{192} m_{0}^{2}\langle\bar{q} q\rangle\left(1-i \frac{m_{q}}{6} \not x\right) \\
& -i g_{s} \int_{0}^{1} d u\left[\frac{\not x}{16 \pi^{2} x^{2}} G_{\mu \nu}(u x) \sigma_{\mu \nu}-\frac{i}{4 \pi^{2} x^{2}} u x^{\mu} G_{\mu \nu}(u x) \gamma^{\nu}\right. \\
& \left.-i \frac{m_{q}}{32 \pi^{2}} G_{\mu \nu}(u x) \sigma^{\mu \nu}\left(\ln \left(\frac{-x^{2} \Lambda^{2}}{4}\right)+2 \gamma_{E}\right)\right]
\end{aligned}
$$

and

$$
\begin{aligned}
S_{Q}(x)= & \frac{m_{Q}^{2}}{4 \pi^{2}} \frac{K_{1}\left(m_{Q} \sqrt{-x^{2}}\right)}{\sqrt{-x^{2}}}-i \frac{m_{Q}^{2} \not x}{4 \pi^{2} x^{2}} K_{2}\left(m_{Q} \sqrt{-x^{2}}\right) \\
& -i g_{s} \int \frac{d^{4} k}{(2 \pi)^{4}} e^{-i k x} \int_{0}^{1} d u\left[\frac{\not k+m_{Q}}{2\left(m_{Q}^{2}-k^{2}\right)^{2}} G^{\mu \nu}(u x) \sigma_{\mu \nu}\right. \\
& \left.+\frac{u}{m_{Q}^{2}-k^{2}} x_{\mu} G^{\mu \nu}(u x) \gamma_{\nu}\right]
\end{aligned}
$$

where $\gamma_{E}$ is the Euler constant, $G_{\mu \nu}$ is the gluon field strength tensor, $\Lambda$ is the scale parameter and $K_{\nu}$ in the heavy propagator denote the Bessel functions of the second kind. By inserting the explicit expressions of the heavy and light propagators we end up with an expression in coordinate space that we need to transfer it to the momentum space in order to make the calculations easy and ready for the application of the Borel transformation as well as the continuum subtraction to enhance the ground state pole contribution and suppress the effects of the higher state and continuum. In the case of heavy systems with more than one heavy quarks, using the integral representation of the Bessel functions of the second kind, appearing in the heavy quark propagator in the form

$$
\frac{K_{\nu}\left(m_{Q} \sqrt{-x^{2}}\right)}{\left(\sqrt{-x^{2}}\right)^{v}}=\frac{1}{2} \int_{0}^{\infty} \frac{d t}{t^{\nu+1}} \exp \left[-\frac{m_{Q}}{2}\left(t-\frac{x^{2}}{t}\right)\right],
$$

in Minkowski space with $m_{Q}$ being the heavy quark mass, leads to a well-known problem and we end up with indeterminate results in the calculations of the continuum subtraction (for details see the Appendix C of the Ref. [4]). By choosing an appropriate representation 
of the Bessel functions and applying successive Borel transformations with the aim of more suppressing the unwanted contributions, we show how we obtain a finite result for systems of multi-heavy quarks. In the following, we present the mathematics required for these processes in the light, single heavy, doubly heavy, triple heavy, four heavy and five heavy systems. Note that we will use the free parts of the quark propagators to present the calculations, however, the applied method is also valid when we include the interacting parts of the quark propagators. The interacting parts of the heavy quark propagator can also be written in terms of the modified Bessel functions [18].

\section{$2.1 \quad$ Light systems}

In the following, we study the mathematics required for investigation of the light hadrons containing the light $(u, d, s)$ quarks and their interaction with the photon and other particles. Inserting the light quarks' propagators into the expression obtained after contracting out the quark pairs in the correlation functions in many non-perturbative methods, we get the following generic expression in $x$-space:

$$
T_{0}(p, q)=\int_{0}^{1} d u \int d^{4} x e^{i P . x} f(u) \frac{1}{\left(-x^{2}\right)^{n}},
$$

where $P=p+u q$ with $u q$ coming from distribution amplitudes of the on-shell state, $u$ is the momentum fraction and $f(u)$ is a general function. In the following for simplicity we omit the dependence of $T_{0}$ function on $p$ and $q$. We perform a Wick rotation to go the Euclidean space

$$
T_{0}=-i \int_{0}^{1} d u \int d^{4} x e^{-i P . x} f(u) \frac{1}{\left(x^{2}\right)^{n}} .
$$

Now we use the Schwinger parametrization

$$
\frac{1}{A^{n}}=\frac{1}{\Gamma(n)} \int_{0}^{\infty} d t t^{n-1} e^{-t A} \quad A>0,
$$

and get

$$
T_{0}=\frac{-i}{\Gamma(n)} \int_{0}^{1} d u \int d^{4} x f(u) \int_{0}^{\infty} d t e^{-i P \cdot x} e^{-t x^{2}} t^{n-1} .
$$

The next step is to make the power of the exponential full-squared and perform the resultant Gaussian integral over four $x$ using

$$
\int d^{4} x e^{-i P \cdot x} e^{-t x^{2}}=\left(\frac{\pi}{t}\right)^{2} e^{\frac{-P^{2}}{4 t}} .
$$

This leads to

$$
T_{0}=\frac{-i}{\Gamma(n)} \int_{0}^{1} d u f(u) \int_{0}^{\infty} d t e^{\frac{-P^{2}}{4 t}}\left(\frac{\pi}{t}\right)^{2} t^{n-1}
$$

Using the double Borel transformation with respect to $(p+q)^{2}$ and $p^{2}$ by the help of

$$
\mathcal{B}_{M_{1}} \mathcal{B}_{M_{2}} e^{\frac{-P^{2}}{4 t}}=\delta\left(\frac{1}{M_{1}^{2}}-\frac{u}{4 t}\right) \delta\left(\frac{1}{M_{2}^{2}}-\frac{\bar{u}}{4 t}\right) e^{\frac{q^{2}}{M_{1}^{2}+M_{2}^{2}}},
$$

and performing the $u$ and $t$ integration, one obtains

$$
\mathcal{B}_{M_{1}} \mathcal{B}_{M_{2}} T_{0}=\frac{-i 4^{2-n} \pi^{2}}{\Gamma(n)} f\left(u_{0}\right)\left(M^{2}\right)^{n} e^{\frac{q^{2}}{M_{1}^{2}+M_{2}^{2}}},
$$

where $u_{0}=\frac{M_{2}^{2}}{M_{1}^{2}+M_{2}^{2}}$ and $M^{2}=\frac{M_{1}^{2} M_{2}^{2}}{M_{1}^{2}+M_{2}^{2}}$. 


\section{Spectral representation and continuum subtraction for light systems}

Our aim is to write the $T_{0}$ function in terms of a double dispersion integral

$$
T_{0}\left((p+q)^{2}, p^{2}\right)=\int_{0}^{\infty} d s_{1} \int_{0}^{\infty} d s_{2} \frac{\rho_{0}\left(s_{1}, s_{2}\right)}{\left[s_{1}-(p+q)^{2}\right]\left[s_{2}-p^{2}\right]},
$$

and find the spectral density $\rho_{0}\left(s_{1}, s_{2}\right)$. The final goal is the application of continuum subtraction in order to more suppress the contributions of higher states and continuum. The double Borel transformed form of the $T_{0}$ function is written as

$$
T_{0}\left(M_{1}^{2}, M_{2}^{2}\right)=\int_{0}^{\infty} d s_{1} \int_{0}^{\infty} d s_{2} \rho_{0}\left(s_{1}, s_{2}\right) e^{-\frac{s_{1}}{M_{1}^{2}}} e^{-\frac{s_{2}}{M_{2}^{2}}}
$$

Now, let us discuss how contributions of the continuum and higher states are subtracted and the spectral density $\rho_{0}\left(s_{1}, s_{2}\right)$ is obtained. To this end, we consider a generic term of the form

$$
\Pi_{0}=\left(M^{2}\right)^{n} f\left(u_{0}\right) .
$$

The first step is to expand $f\left(u_{0}\right)$ as

$$
f\left(u_{0}\right)=\sum_{k} a_{k} u_{0}^{k}
$$

As a result we get

$$
\Pi_{0}=\left(\frac{M_{1}^{2} M_{2}^{2}}{M_{1}^{2}+M_{2}^{2}}\right)^{n} \sum_{k} a_{k}\left(\frac{M_{2}^{2}}{M_{1}^{2}+M_{2}^{2}}\right)^{k} .
$$

Introducing new variables, $\sigma_{i}=\frac{1}{M_{i}^{2}}$, we have

$$
\begin{aligned}
\Pi_{0} & =\sum_{k} a_{k} \frac{\sigma_{1}^{k}}{\left(\sigma_{1}+\sigma_{2}\right)^{n+k}} \\
& =\sum_{k} a_{k} \frac{\sigma_{1}^{k}}{\Gamma(n+k)} \int_{0}^{\infty} d t e^{-t\left(\sigma_{1}+\sigma_{2}\right)} t^{n+k-1}
\end{aligned}
$$

Applying double Borel transformation with respect to $\sigma_{1} \rightarrow \frac{1}{\tau_{1}}$ and $\sigma_{2} \rightarrow \frac{1}{\tau_{2}}$, we obtain the spectral density as

$$
\mathcal{B}_{1 / \tau_{1}} \mathcal{B}_{1 / \tau_{2}} \Pi_{0}=\sum_{k} a_{k} \frac{(-1)^{k}}{\Gamma(n+k)} \int_{0}^{\infty} d t t^{n+k-1}\left(\left(\frac{d}{d t}\right)^{k} \delta\left(\tau_{1}-t\right)\right) \delta\left(\tau_{1}-t\right) .
$$

We define the spectral density $\rho_{0}\left(s_{1}, s_{2}\right)=\mathcal{B}_{1 / \tau_{1}} \mathcal{B}_{1 / \tau_{2}} \Pi_{0}$ with $\tau_{1} \rightarrow s_{1}$ and $\tau_{2} \rightarrow s_{2}$. Hence,

$$
\rho_{0}\left(s_{1}, s_{2}\right)=\sum_{k} a_{k} \frac{(-1)^{k}}{\Gamma(n+k)} \int_{0}^{\infty} d t t^{n+k-1}\left(\left(\frac{d}{d t}\right)^{k} \delta\left(s_{1}-t\right)\right) \delta\left(s_{2}-t\right) .
$$

Performing integration over $t$, finally, we obtain the following expression for the double spectral density:

$$
\rho_{0}\left(s_{1}, s_{2}\right)=\sum_{k} a_{k} \frac{(-1)^{k}}{\Gamma(n+k)} s_{1}^{n+k-1} \delta^{k}\left(s_{2}-s_{1}\right),
$$


where $\delta^{k}(x)=\frac{d^{k}}{d x^{k}} \delta(x)$. Using this spectral density, the continuum subtracted correlation function in the Borel scheme corresponding to the considered term can be written as:

$$
\Pi_{0}^{s u b}\left(M_{1}^{2}, M_{2}^{2}\right)=\int_{0}^{s_{1}} d s_{1} \int_{0}^{s_{2}} d s_{2} \rho\left(s_{1}, s_{2}\right) e^{-s_{1} / M_{1}^{2}} e^{-s_{2} / M_{2}^{2}},
$$

where "sub" stands for subtracted. Defining new variables, $s_{1}=s v$ and $s_{2}=s(1-v)$, we get

$$
\Pi_{0}^{s u b}\left(M_{1}^{2}, M_{2}^{2}\right)=\int_{0}^{s_{0}} d s \int_{0}^{1} d v \rho\left(s_{1}, s_{2}\right) s e^{-s v / M_{1}^{2}} e^{-s(1-v) / M_{2}^{2}} .
$$

Finally, using the expression for the spectral density, one can get

$$
\Pi_{0}^{s u b}\left(M_{1}^{2}, M_{2}^{2}\right)=\sum_{k} a_{k} \frac{(-1)^{k}}{\Gamma(n+k)} \int_{0}^{s_{0}} d s \frac{s^{n-1}}{2}\left(\frac{d}{d v}\right)^{k}\left[v^{n+k-1} e^{-s v / M_{1}^{2}} e^{-s(1-v) / M_{2}^{2}}\right]_{v=1 / 2} .
$$

\subsection{Systems with one heavy quark}

In the following, we study the hadrons containing a single heavy quark with some light quarks. Let us consider the following generic term:

$$
T_{1}=\int_{0}^{1} d u \int d^{4} x e^{i P . x} f(u) \frac{K_{\nu}\left(m_{Q} \sqrt{-x^{2}}\right)}{\left(\sqrt{-x^{2}}\right)^{n}},
$$

where $m_{Q}$ is the mass of heavy quark and $K_{\nu}$ is the modified Bessel function of the second kind in order $\nu$. The $K_{\nu}$ function comes from the $x$-representation of the heavy quark propagator. Using the following integral representation of the modified Bessel function

$$
K_{\nu}\left(m_{Q} \sqrt{-x^{2}}\right)=\frac{\Gamma(\nu+1 / 2) 2^{\nu}}{\sqrt{\pi} m_{Q}^{\nu}} \int_{0}^{\infty} d t \cos \left(m_{Q} t\right) \frac{\left(\sqrt{-x^{2}}\right)^{\nu}}{\left(t^{2}-x^{2}\right)^{\nu+1 / 2}}
$$

we have

$$
T_{1}=\frac{\Gamma(\nu+1 / 2) 2^{\nu}}{\sqrt{\pi} m_{Q}^{\nu}} \int_{0}^{1} d u \int d^{4} x e^{i P \cdot x} f(u) \int_{0}^{\infty} d t \frac{\cos \left(m_{Q} t\right)}{\left(t^{2}-x^{2}\right)^{\nu+1 / 2}\left(x^{2}\right)^{\frac{n-\nu}{2}}} .
$$

We perform a Wick rotation to go to the Euclidean space

$$
T_{1}=\frac{\Gamma(\nu+1 / 2) 2^{\nu}}{\sqrt{\pi} m_{Q}^{\nu}} \int_{0}^{1} d u \int d^{4} x(-i) e^{-i P . x} f(u) \int_{0}^{\infty} d t \frac{\cos \left(m_{Q} t\right)}{\left(t^{2}+x^{2}\right)^{\nu+1 / 2}\left(x^{2}\right)^{\frac{n-\nu}{2}}} .
$$

Using the identify

$$
\frac{1}{A^{n}}=\frac{1}{\Gamma(n)} \int_{0}^{\infty} d t t^{n-1} e^{-t A} \quad A>0,
$$

Eq. (30) can be reformulated as

$$
\begin{aligned}
T_{1} & =\frac{2^{\nu}(-i)}{\Gamma\left(\frac{n-\nu}{2}\right) \sqrt{\pi} m_{Q}^{\nu}} \int_{0}^{1} d u \int d^{4} x e^{-i P . x} f(u) \\
& \times \int_{0}^{\infty} d t \int_{0}^{\infty} d y \int_{0}^{\infty} d v v^{\nu-\frac{1}{2}} e^{-v\left(x^{2}+t^{2}\right)} y^{\frac{n-\nu}{2}-1} e^{-y\left(x^{2}\right)} \cos \left(m_{Q} t\right)
\end{aligned}
$$


In the next step, we performe the Gaussian integral over four- $x$ and $t$

$$
\int d^{4} x e^{-i P \cdot x} e^{-v x^{2}} e^{-y x^{2}}=\left(\frac{\pi}{y+v}\right)^{2} e^{\frac{-P^{2}}{4(y+v)}},
$$

and

$$
\int_{0}^{\infty} d t \cos (m t) e^{-v t^{2}}=\frac{e^{\frac{-m^{2}}{4 v}} \sqrt{\pi}}{2 \sqrt{v}}
$$

As a result, we get:

$$
T_{1}=\frac{2^{\nu-1} \pi^{2}(-i)}{\Gamma\left(\frac{n-\nu}{2}\right) m_{Q}^{\nu}} \int_{0}^{1} d u f(u) \int_{0}^{\infty} d y \int_{0}^{\infty} d v \frac{e^{\frac{-m^{2}}{4 v}} v^{\nu-1} y^{\frac{n-\nu}{2}-1} e^{\frac{-P^{2}}{4(y+v)}}}{(v+y)^{2}} .
$$

We introduce the new variables $\rho$ and $\lambda$, defined by

$$
\rho=v+y, \quad \lambda=\frac{y}{v+y}
$$

which leads to

$$
T_{1}=\frac{2^{\nu-1} \pi^{2}(-i)}{\Gamma\left(\frac{n-\nu}{2}\right) m_{Q}^{\nu}} \int_{0}^{1} d u f(u) \int_{0}^{\infty} d \rho \int_{0}^{1} d \lambda e^{\frac{-m^{2}}{4 \rho(1-\lambda)}} \rho^{\frac{n+\nu}{2}-3}(1-\lambda)^{\nu-1} \lambda^{\frac{n-\nu}{2}-1} e^{\frac{-P^{2}}{4 \rho}} .
$$

Using the Borel transformation of the exponential

$$
\mathcal{B}_{M_{1}} \mathcal{B}_{M_{2}} e^{\frac{-P^{2}}{4 \rho}}=\delta\left(\frac{1}{M_{1}^{2}}-\frac{u}{4 \rho}\right) \delta\left(\frac{1}{M_{2}^{2}}-\frac{\bar{u}}{4 \rho}\right) e^{\frac{q^{2}}{M_{1}^{2}+M_{2}^{2}}},
$$

and performing the $u$ and $\rho$ integrals, one obtain

$$
\mathcal{B}_{M_{1}} \mathcal{B}_{M_{2}} T_{1}=-i \frac{2^{2-n} \pi^{2}}{m_{Q}^{\nu}} f\left(u_{0}\right)\left(M^{2}\right)^{\frac{\nu+n}{2}} \int_{0}^{1} d \lambda \frac{e^{\frac{-m^{2}}{M^{2}(1-\lambda)}}(1-\lambda)^{\nu-1} \lambda^{\frac{n-\nu}{2}-1}}{\Gamma\left(\frac{n-\nu}{2}\right)} e^{\frac{q^{2}}{M_{1}^{2}+M_{2}^{2}}}
$$

where $u_{0}=\frac{M_{2}^{2}}{M_{1}^{2}+M_{2}^{2}}$ and $M^{2}=\frac{M_{1}^{2} M_{2}^{2}}{M_{1}^{2}+M_{2}^{2}}$. By replacing $\lambda=x^{2}$, we get

$$
\mathcal{B}_{M_{1}} \mathcal{B}_{M_{2}} T_{1}=-i \frac{2^{2-n} \pi^{2}}{m_{Q}^{\nu}} f\left(u_{0}\right)\left(M^{2}\right)^{\frac{\nu+n}{2}} e^{\frac{q^{2}}{M_{1}^{2}+M_{2}^{2}}} \int_{0}^{1} d x \frac{e^{\frac{-m^{2}}{M^{2}\left(1-x^{2}\right)}}\left(1-x^{2}\right)^{\nu-1} x^{n-\nu-1}}{\Gamma\left(\frac{n-\nu}{2}\right)} .
$$

In the last step, we change the variable $\frac{1}{1-x^{2}} \rightarrow t$ and we get

$$
\mathcal{B}_{M_{1}} \mathcal{B}_{M_{2}} T_{1}=-i \frac{2^{2-n} \pi^{2}}{m_{Q}^{\nu}} f\left(u_{0}\right)\left(M^{2}\right)^{\frac{\nu+n}{2}} e^{\frac{q^{2}}{M_{1}^{2}+M_{2}^{2}}} \psi\left(\alpha, \beta, \frac{m_{Q}^{2}}{M^{2}}\right)
$$

where

$$
\psi\left(\alpha, \beta, \frac{m_{Q}^{2}}{M^{2}}\right)=\frac{1}{\Gamma(\alpha)} \int_{1}^{\infty} d t e^{-t \frac{m_{Q}^{2}}{M^{2}} t^{\beta-\alpha-1}(t-1)^{\alpha-1},}
$$

with $\alpha=\frac{n-\nu}{2}$ and $\beta=1-\nu$. 


\section{Spectral representation and continuum subtraction for systems containing one heavy quark}

Now, let us discuss how contribution of the continuum and higher states are subtracted. As is obvious from Eq. (41), the generic term has the form

$$
\Pi_{1}=\mathcal{C}\left(M^{2}\right)^{\frac{\nu+n}{2}} f\left(u_{0}\right) \psi\left(\alpha, \beta, \frac{m_{Q}^{2}}{M^{2}}\right),
$$

where

$$
\mathcal{C}=-i \frac{2^{2-n} \pi^{2}}{m_{Q}^{\nu}}
$$

The first step again is to expand $f\left(u_{0}\right)$ as

$$
f\left(u_{0}\right)=\sum_{k} a_{k} u_{0}^{k} .
$$

As a result we get

$$
\Pi_{1}=\mathcal{C}\left(\frac{M_{1}^{2} M_{2}^{2}}{M_{1}^{2}+M_{2}^{2}}\right)^{\frac{\nu+n}{2}} \sum_{k} a_{k}\left(\frac{M_{2}^{2}}{M_{1}^{2}+M_{2}^{2}}\right)^{k} \frac{1}{\Gamma(\alpha)} \int_{1}^{\infty} d t e^{-t \frac{m_{Q}^{2}}{M^{2}} t^{\beta-\alpha-1}(t-1)^{\alpha-1} .}
$$

Introducing new variables, $\sigma_{i}=\frac{1}{M_{i}^{2}}$, we have

$$
\begin{aligned}
\Pi_{1} & =\mathcal{C} \sum_{k} a_{k} \frac{\sigma_{1}^{k}}{\left(\sigma_{1}+\sigma_{2}\right)^{\frac{\nu+n}{2}+k}} \frac{1}{\Gamma(\alpha)} \int_{1}^{\infty} d t e^{-t m_{Q}^{2}\left(\sigma_{1}+\sigma_{2}\right)} t^{\beta-\alpha-1}(t-1)^{\alpha-1} \\
& =\mathcal{C} \sum_{k} a_{k} \frac{\sigma_{1}^{k}}{\Gamma\left(\frac{\nu+n}{2}+k\right) \Gamma(\alpha)} \int_{1}^{\infty} d t e^{-t m_{Q}^{2}\left(\sigma_{1}+\sigma_{2}\right)} t^{\beta-\alpha-1}(t-1)^{\alpha-1} \int_{0}^{\infty} d l e^{-l\left(\sigma_{1}+\sigma_{2}\right)} l^{\frac{\nu+n}{2}+k-1} \\
& =\mathcal{C} \sum_{k} a_{k} \frac{\sigma_{1}^{k}}{\Gamma\left(\frac{\nu+n}{2}+k\right) \Gamma(\alpha)} \int_{1}^{\infty} d t t^{\beta-\alpha-1}(t-1)^{\alpha-1} \int_{0}^{\infty} d l l^{\frac{\nu+n}{2}+k-1} e^{-\left(l+t m_{Q}^{2}\right)\left(\sigma_{1}+\sigma_{2}\right)} \\
& =\mathcal{C} \sum_{k} a_{k} \frac{(-1)^{k}}{\Gamma\left(\frac{\nu+n}{2}+k\right) \Gamma(\alpha)} \int_{1}^{\infty} d t t^{\beta-\alpha-1}(t-1)^{\alpha-1} \\
& \times \int_{0}^{\infty} d l l^{\frac{\nu+n}{2}+k-1}\left(\left(\frac{d}{d l}\right)^{k} e^{-\left(l+t m_{Q}^{2}\right) \sigma_{1}}\right) e^{-\left(l+t m_{Q}^{2}\right) \sigma_{2}} .
\end{aligned}
$$

Applying double Borel transformation with respect to $\sigma_{1} \rightarrow \frac{1}{\tau_{1}}$ and $\sigma_{2} \rightarrow \frac{1}{\tau_{2}}$, we obtain

$$
\begin{aligned}
\mathcal{B}_{1 / \tau_{1}} \mathcal{B}_{1 / \tau_{2}} \Pi_{1} & =\mathcal{C} \sum_{k} a_{k} \frac{(-1)^{k}}{\Gamma\left(\frac{\nu+n}{2}+k\right) \Gamma(\alpha)} \int_{1}^{\infty} d t t^{\beta-\alpha-1}(t-1)^{\alpha-1} \int_{0}^{\infty} d l l^{\frac{\nu+n}{2}+k-1} \\
& \times\left(\left(\frac{d}{d l}\right)^{k} \delta\left(\tau_{1}-\left(l+t m_{Q}^{2}\right)\right)\right) \delta\left(\tau_{2}-\left(l+t m_{Q}^{2}\right)\right) .
\end{aligned}
$$

The double spectral density $\rho_{1}\left(s_{1}, s_{2}\right)$ is found by replacing $\tau_{1} \rightarrow s_{1}$ and $\tau_{2} \rightarrow s_{2}$ in the expression as

$$
\begin{aligned}
\rho_{1}\left(s_{1}, s_{2}\right) & =\mathcal{C} \sum_{k} a_{k} \frac{(-1)^{k}}{\Gamma\left(\frac{\nu+n}{2}+k\right) \Gamma(\alpha)} \int_{1}^{\infty} d t t^{\beta-\alpha-1}(t-1)^{\alpha-1} \int_{0}^{\infty} d l l^{\frac{\nu+n}{2}+k-1} \\
& \times\left(\left(\frac{d}{d l}\right)^{k} \delta\left(s_{1}-\left(l+t m_{Q}^{2}\right)\right)\right) \delta\left(s_{2}-\left(l+t m_{Q}^{2}\right)\right) .
\end{aligned}
$$


Performing the integration over $l$, finally we obtain the following expression for the double spectral density:

$$
\begin{aligned}
\rho_{1}\left(s_{1}, s_{2}\right) & =\mathcal{C} \sum_{k} a_{k} \frac{(-1)^{k}}{\Gamma\left(\frac{\nu+n}{2}+k\right) \Gamma(\alpha)} \int_{1}^{\infty} d t t^{\beta-\alpha-1}(t-1)^{\alpha-1}\left(s_{1}-t m_{Q}^{2}\right)^{\frac{\nu+n}{2}+k-1} \\
& \times\left(\left(\frac{d}{d s_{1}}\right)^{k} \delta\left(s_{2}-s_{1}\right)\right) \theta\left(s_{1}-t m_{Q}^{2}\right),
\end{aligned}
$$

or

$$
\begin{aligned}
\rho_{1}\left(s_{1}, s_{2}\right) & =\mathcal{C} \sum_{k} a_{k} \frac{(-1)^{k}}{\Gamma\left(\frac{\nu+n}{2}+k\right) \Gamma(\alpha)} \int_{1}^{s_{1} / m_{Q}^{2}} d t t^{\beta-\alpha-1}(t-1)^{\alpha-1}\left(s_{1}-t m_{Q}^{2}\right)^{\frac{\nu+n}{2}+k-1} \\
& \times\left(\left(\frac{d}{d s_{1}}\right)^{k} \delta\left(s_{2}-s_{1}\right)\right) .
\end{aligned}
$$

Using this spectral density, the continuum subtracted correlation function in the Borel scheme corresponding to the considered term can be written as:

$$
\Pi^{s u b}\left(M_{1}^{2}, M_{2}^{2}\right)=\int_{m_{Q}^{2}}^{s_{0}} d s_{1} \int_{m_{Q}^{2}}^{s_{0}} d s_{2} \rho\left(s_{1}, s_{2}\right) e^{-s_{1} / M_{1}^{2}} e^{-s_{2} / M_{2}^{2}} .
$$

Defining new variables, $s_{1}=2 s v$ and $s_{2}=2 s(1-v)$, we get

$$
\Pi^{s u b}\left(M_{1}^{2}, M_{2}^{2}\right)=\int_{m_{Q}^{2}}^{s_{0}} d s \int_{0}^{1} d v \rho\left(s_{1}, s_{2}\right)(4 s) e^{-2 s v / M_{1}^{2}} e^{-2 s(1-v) / M_{2}^{2}} .
$$

Using the expression for the spectral density, one can get

$$
\begin{aligned}
\Pi_{1}^{s u b}\left(M_{1}^{2}, M_{2}^{2}\right) & =\mathcal{C} \sum_{k} a_{k} \frac{(-1)^{k}}{\Gamma\left(\frac{\nu+n}{2}+k\right) \Gamma(\alpha)} \int_{m_{Q}^{2}}^{s_{0}} d s \int_{0}^{1} d v \frac{1}{2^{k} s^{k}}\left(\left(\frac{d}{d v}\right)^{k} \delta(v-1 / 2)\right) \\
& \times \int_{1}^{2 s v / m_{Q}^{2}} d t t^{\beta-\alpha-1}(t-1)^{\alpha-1}\left(2 s v-t m_{Q}^{2}\right)^{\frac{\nu+n}{2}+k-1} e^{-2 s v / M_{1}^{2}} e^{-2 s(1-v) / M_{2}^{2}} .
\end{aligned}
$$

By integrating by part over $v$, finally we obtain

$$
\begin{aligned}
\Pi_{1}^{s u b}\left(M_{1}^{2}, M_{2}^{2}\right) & =-i \sum_{k} a_{k} \frac{2^{2-n} \pi^{2}}{m_{Q}^{\nu} \Gamma\left(\frac{\nu+n}{2}+k\right) \Gamma(\alpha)} \int_{m_{Q}^{2}}^{s_{0}} d s \frac{1}{2^{k} s^{k}}\left[\left(\frac{d}{d v}\right)^{k} \int_{1}^{2 s v / m_{Q}^{2}} d t t^{\beta-\alpha-1}(t-1)^{\alpha-1}\right. \\
& \left.\times\left(2 s v-t m_{Q}^{2}\right)^{\frac{\nu+n}{2}+k-1} e^{-2 s v / M_{1}^{2}} e^{-2 s(1-v) / M_{2}^{2}}\right]_{v=1 / 2} .
\end{aligned}
$$

\subsection{Systems containing two heavy quarks}

In the following, we study hadrons containing two heavy quarks and some light quarks. Let us consider again the following generic term:

$$
T_{2}=\int_{0}^{1} d u \int d^{4} x e^{i P . x} A(u) \frac{K_{\nu}\left(m_{1 Q} \sqrt{-x^{2}}\right) K_{\mu}\left(m_{2 Q} \sqrt{-x^{2}}\right)}{\left(\sqrt{-x^{2}}\right)^{n}},
$$


where $m_{i Q}$ 's are the masses of heavy quarks and $K_{\nu}$ and $K_{\mu}$ are the modified Bessel functions of order $\nu$ and $\mu$, respectively. Using the integral representation of the modified Bessel functions from Eq. (28), we have

$$
\begin{aligned}
T_{2} & =\frac{\Gamma(\nu+1 / 2) \Gamma(\mu+1 / 2) 2^{\mu+\nu}}{\pi m_{1 Q}^{\nu} m_{2 Q}^{\mu}} \int_{0}^{1} d u \int d^{4} x e^{i P . x} A(u) \\
& \times \int_{0}^{\infty} d t_{2} \int_{0}^{\infty} d t_{1} \frac{\cos \left(m_{1 Q} t_{1}\right) \cos \left(m_{2 Q} t_{2}\right)}{\left(t_{1}^{2}-x^{2}\right)^{\nu+1 / 2}\left(t_{2}^{2}-x^{2}\right)^{\mu+1 / 2}\left(x^{2}\right)^{\frac{n-\nu-\mu}{2}}} .
\end{aligned}
$$

We again perform a Wick rotation to go the Euclidean space

$$
\begin{aligned}
T_{2} & =(-i) \frac{\Gamma(\nu+1 / 2) \Gamma(\mu+1 / 2) 2^{\mu+\nu}}{\pi m_{1 Q}^{\nu} m_{2 Q}^{\mu}} \int_{0}^{1} d u \int d^{4} x e^{-i P . x} A(u) \\
& \times \int_{0}^{\infty} d t_{2} \int_{0}^{\infty} d t_{1} \frac{\cos \left(m_{1 Q} t_{1}\right) \cos \left(m_{2 Q} t_{2}\right)}{\left(t_{1}^{2}+x^{2}\right)^{\nu+1 / 2}\left(t_{2}^{2}+x^{2}\right)^{\mu+1 / 2}\left(x^{2}\right)^{\frac{n-\nu-\mu}{2}}} .
\end{aligned}
$$

Using the Schwinger integral representation presented in Eq. (31), Eq. (158) can be reformulated as

$$
\begin{aligned}
T_{2} & =(-i) \frac{2^{\mu+\nu}}{\pi m_{1 Q}^{\nu} m_{2 Q}^{\mu} \Gamma\left(\frac{n-\nu-\mu}{2}\right)} \int_{0}^{1} d u \int d^{4} x e^{-i P . x} A(u) \int_{0}^{\infty} d t_{2} \int_{0}^{\infty} d t_{1} \cos \left(m_{1 Q} t_{1}\right) \cos \left(m_{2 Q} t_{2}\right) \\
& \times \int_{0}^{\infty} d y_{2} \int_{0}^{\infty} d y_{1} \int_{0}^{\infty} d y_{0} y_{0}^{\left(\frac{n-\nu-\mu}{2}\right)-1} e^{-y_{0} x^{2}} y_{1}^{\nu-\frac{1}{2}} e^{-y_{1}\left(x^{2}+t_{1}^{2}\right)} y_{2}^{\mu-\frac{1}{2}} e^{-y_{2}\left(x^{2}+t_{2}^{2}\right)} .
\end{aligned}
$$

Performing the Gaussian integral over four- $x, t_{1}$ and $t_{2}$, we get

$$
\begin{aligned}
T_{2} & =(-i) \frac{2^{\mu+\nu-2} \pi^{2}}{m_{1 Q}^{\nu} m_{2 Q}^{\mu} \Gamma\left(\frac{n-\nu-\mu}{2}\right)} \int_{0}^{1} d u A(u) \int_{0}^{\infty} d y_{0} y_{0}^{\left(\frac{n-\nu-\mu}{2}\right)-1} \\
& \times \int_{0}^{\infty} d y_{2} \int_{0}^{\infty} d y_{1} \frac{y_{1}^{\nu-1} e^{-\frac{m_{1 Q}^{2}}{4 y_{1}}} y_{2}^{\mu-1} e^{-\frac{m_{2 Q}^{2}}{4 y_{2}}} e^{\frac{-P^{2}}{4\left(y_{0}+y_{1}+y_{2}\right)}}}{\left(y_{0}+y_{1}+y_{2}\right)^{2}} .
\end{aligned}
$$

Now we introduce the variables $\rho, v$ and $u$, defined by

$$
\rho=y_{0}+y_{1}+y_{2}, \quad v=\frac{y_{1}}{y_{0}+y_{1}+y_{2}}, \quad w=\frac{y_{2}}{y_{0}+y_{1}+y_{2}},
$$

which leads to

$$
\begin{aligned}
T_{2} & =(-i) \frac{2^{\mu+\nu-2} \pi^{2}}{m_{1 Q}^{\nu} m_{2 Q}^{\mu} \Gamma\left(\frac{n-\nu-\mu}{2}\right)} \int_{0}^{1} d u A(u) \int_{0}^{1} d w(\rho(1-v-w))^{\left(\frac{n-\nu-\mu}{2}\right)-1} \\
& \times \int_{0}^{\infty} d \rho \int_{0}^{1} d v(\rho v)^{\nu-1} e^{-\frac{m_{1 Q}^{2}}{4 \rho v}}(\rho w)^{\mu-1} e^{-\frac{m_{2 Q}^{2}}{4 \rho w}} e^{\frac{-P^{2}}{4 \rho}}
\end{aligned}
$$

Applying again the double Borel transformations with respect to $(p+q)^{2} \rightarrow M_{1}^{2}$ and $p^{2} \rightarrow M_{2}^{2}$ and performing the integrals over the variables $u$ and $\rho$, using the resultant Dirac Deltas, we obtain 


$$
\begin{aligned}
\mathcal{B}_{M_{1}} \mathcal{B}_{M_{2}} T_{2} & =(-i) \frac{2^{2-n} \pi^{2}}{m_{1 Q}^{\nu} m_{2 Q}^{\mu} \Gamma\left(\frac{n-\nu-\mu}{2}\right)} A\left(u_{0}\right)\left(M^{2}\right)^{\frac{n+\nu+\mu}{2}} e^{\frac{q^{2}}{M_{1}^{2}+M_{2}^{2}}} \int_{0}^{1} d w \int_{0}^{1} d v \\
& \times(1-v-w)^{\left(\frac{n-\nu-\mu}{2}\right)-1} e^{-\frac{m_{1 Q}^{2}}{M^{2} v}} v^{\nu-1} e^{-\frac{m_{2 Q}^{2}}{M^{2} w}}(w)^{\mu-1} .
\end{aligned}
$$

The new variables

$$
v=z(1-y), \quad w=z y,
$$

change the above expression to

$$
\begin{aligned}
\mathcal{B}_{M_{1}} \mathcal{B}_{M_{2}} T_{2} & =(-i) \frac{2^{2-n} \pi^{2}}{m_{1 Q}^{\nu} m_{2 Q}^{\mu} \Gamma\left(\frac{n-\nu-\mu}{2}\right)} A\left(u_{0}\right)\left(M^{2}\right)^{\frac{n+\nu+\mu}{2}} e^{\frac{q^{2}}{M_{1}^{2}+M_{2}^{2}}} \int_{0}^{2} d z \int_{0}^{1} d y \\
& \times z^{\nu+\mu-1}(1-z)^{\left(\frac{n-\nu-\mu}{2}\right)-1}(1-y)^{\nu-1} y^{\mu-1} e^{-\frac{m_{1 Q}^{2}}{M^{2} z(1-y)}} e^{-\frac{m_{2 Q}^{2}}{M^{2} z y}}
\end{aligned}
$$

Spectral representation and continuum subtraction for systems containing two heavy quarks

Now, let us discuss how contributions of the higher states and continuum are subtracted. We consider again a generic term of the form

$$
\begin{aligned}
\Pi_{2} & =\mathcal{C}\left(M^{2}\right)^{\frac{n+\nu+\mu}{2}} A\left(u_{0}\right) \int_{0}^{2} d z \int_{0}^{1} d y z^{\nu+\mu-1}(1-z)^{\left(\frac{n-\nu-\mu}{2}\right)-1} \\
& \times(1-y)^{\nu-1} y^{\mu-1} e^{-\frac{m_{1 Q}^{2}}{M^{2} z(1-y)}} e^{-\frac{m_{2 Q}^{2}}{M^{2} z y}}
\end{aligned}
$$

where

$$
\mathcal{C}=(-i) \frac{2^{2-n} \pi^{2}}{m_{1 Q}^{\nu} m_{2 Q}^{\mu} \Gamma\left(\frac{n-\nu-\mu}{2}\right)} .
$$

The first step is to expand $A\left(u_{0}\right)$ as

$$
A\left(u_{0}\right)=\sum_{k} a_{k} u_{0}^{k} .
$$

As a result we get

$$
\begin{aligned}
\Pi_{2} & =\mathcal{C}\left(\frac{M_{1}^{2} M_{2}^{2}}{M_{1}^{2}+M_{2}^{2}}\right)^{\frac{n+\nu+\mu}{2}} \sum_{k} a_{k}\left(\frac{M_{2}^{2}}{M_{1}^{2}+M_{2}^{2}}\right)^{k} \int_{0}^{2} d z \int_{0}^{1} d y \\
& \times z^{\nu+\mu-1}(1-z)^{\left(\frac{n-\nu-\mu}{2}\right)-1}(1-y)^{\nu-1} y^{\mu-1} e^{-\frac{m_{1 Q}^{2}}{M^{2} z(1-y)}} e^{-\frac{m_{2 Q}^{2}}{M^{2} z y}}
\end{aligned}
$$


Introducing new variables, $\sigma_{i}=\frac{1}{M_{i}^{2}}$, we have

$$
\begin{aligned}
\Pi_{2}= & \mathcal{C} \sum_{k} a_{k} \frac{\sigma_{1}^{k}}{\left(\sigma_{1}+\sigma_{2}\right)^{\frac{n+\nu+\mu}{2}+k}} \int_{0}^{2} d z \int_{0}^{1} d y z^{\nu+\mu-1}(1-z)^{\left(\frac{n-\nu-\mu}{2}\right)-1} \\
\times & (1-y)^{\nu-1} y^{\mu-1} e^{-\frac{m_{1 Q}^{2}\left(\sigma_{1}+\sigma_{2}\right)}{z(1-y)}} e^{-\frac{m_{2 Q}^{2}\left(\sigma_{1}+\sigma_{2}\right)}{z y}} \\
= & \mathcal{C} \sum_{k} a_{k} \frac{\sigma_{1}^{k}}{\Gamma\left(\frac{n+\nu+\mu}{2}+k\right)} \int_{0}^{2} d z \int_{0}^{1} d y z^{\nu+\mu-1}(1-z)^{\left(\frac{n-\nu-\mu}{2}\right)-1} \\
\times & (1-y)^{\nu-1} y^{\mu-1} e^{-\frac{m_{1 Q}^{2}\left(\sigma_{1}+\sigma_{2}\right)}{z(1-y)}} e^{-\frac{m_{2 Q}^{2}\left(\sigma_{1}+\sigma_{2}\right)}{z y}} \int_{0}^{\infty} d l e^{-l\left(\sigma_{1}+\sigma_{2}\right)} l^{\frac{n+\nu+\mu}{2}+k-1} \\
= & \mathcal{C} \sum_{k} a_{k} \frac{\sigma_{1}^{k}}{\Gamma\left(\frac{n+\nu+\mu}{2}+k\right)} \int_{0}^{2} d z \int_{0}^{1} d y z^{\nu+\mu-1}(1-z)^{\left(\frac{n-\nu-\mu}{2}\right)-1} \\
\times & (1-y)^{\nu-1} y^{\mu-1} \int_{0}^{\infty} d l l^{\frac{n+\nu+\mu}{2}+k-1} e^{-\left(l+\frac{m_{1 Q}^{2}}{z(1-y)}+\frac{m_{2 Q}^{2}}{z y}\right)\left(\sigma_{1}+\sigma_{2}\right)} \\
= & \mathcal{C} \sum_{k} a_{k} \frac{(-1)^{k}}{\Gamma\left(\frac{n+\nu+\mu}{2}+k\right)} \int_{0}^{2} d z \int_{0}^{1} d y z^{\nu+\mu-1}(1-z)^{\left(\frac{n-\nu-\mu}{2}\right)-1} \\
& (1-y)^{\nu-1} y^{\mu-1} \int_{0}^{\infty} d l l^{\frac{n+\nu+\mu}{2}+k-1}\left(\left(\frac{d}{d l}\right)^{k} e^{-\left(l+\frac{m_{1 Q}^{2}}{z(1-y)}+\frac{m_{2 Q}^{2}}{z y}\right) \sigma_{1}}\right) e^{-\left(l+\frac{m_{1 Q}^{2}}{z(1-y)}+\frac{m_{2 Q}^{2}}{z y}\right) \sigma_{2}} .
\end{aligned}
$$

Applying double Borel transformation with respect to $\sigma_{1} \rightarrow \frac{1}{\tau_{1}}$ and $\sigma_{2} \rightarrow \frac{1}{\tau_{2}}$, we obtain the spectral density

$$
\begin{aligned}
\mathcal{B}_{1 / \tau_{1}} \mathcal{B}_{1 / \tau_{2}} \Pi_{2} & =\mathcal{C} \sum_{k} a_{k} \frac{(-1)^{k}}{\Gamma\left(\frac{n+\nu+\mu}{2}+k\right)} \int_{0}^{2} d z \int_{0}^{1} d y z^{\nu+\mu-1} \\
& \times(1-z)^{\left(\frac{n-\nu-\mu}{2}\right)-1}(1-y)^{\nu-1} y^{\mu-1} \int_{0}^{\infty} d l l^{\frac{n+\nu+\mu}{2}+k-1} \\
& \times\left(\left(\frac{d}{d l}\right)^{k} \delta\left(\tau_{1}-\left(l+\frac{m_{1 Q}^{2}}{z(1-y)}+\frac{m_{2 Q}^{2}}{z y}\right)\right) \delta\left(\tau_{2}-\left(l+\frac{m_{1 Q}^{2}}{z(1-y)}+\frac{m_{2 Q}^{2}}{z y}\right)\right),\right.
\end{aligned}
$$

where the spectral density $\rho_{2}\left(s_{1}, s_{2}\right)$ is found by $\tau_{1} \rightarrow s_{1}$ and $\tau_{2} \rightarrow s_{2}$ in this relation

$$
\begin{aligned}
\rho_{2}\left(s_{1}, s_{2}\right) & =\mathcal{C} \sum_{k} a_{k} \frac{(-1)^{k}}{\Gamma\left(\frac{n+\nu+\mu}{2}+k\right)} \int_{0}^{2} d z \int_{0}^{1} d y z^{\nu+\mu-1} \\
& \times(1-z)^{\left(\frac{n-\nu-\mu}{2}\right)-1}(1-y)^{\nu-1} y^{\mu-1} \int_{0}^{\infty} d l l^{\frac{n+\nu+\mu}{2}+k-1} \\
& \times\left(\left(\frac{d}{d l}\right)^{k} \delta\left(s_{1}-\left(l+\frac{m_{1 Q}^{2}}{z(1-y)}+\frac{m_{2 Q}^{2}}{z y}\right)\right) \delta\left(s_{2}-\left(l+\frac{m_{1 Q}^{2}}{z(1-y)}+\frac{m_{2 Q}^{2}}{z y}\right)\right) .\right.
\end{aligned}
$$

Performing integration over $l$, finally we obtain the following expression for the double 
spectral density:

$$
\begin{aligned}
\rho_{2}\left(s_{1}, s_{2}\right) & =\mathcal{C} \sum_{k} a_{k} \frac{(-1)^{k}}{\Gamma\left(\frac{n+\nu+\mu}{2}+k\right)} \int_{0}^{2} d z \int_{0}^{1} d y z^{\nu+\mu-1} \\
& \times(1-z)^{\left(\frac{n-\nu-\mu}{2}\right)-1}(1-y)^{\nu-1} y^{\mu-1}\left(s_{1}-\frac{m_{1 Q}^{2}}{z(1-y)}-\frac{m_{2 Q}^{2}}{z y}\right)^{\frac{n+\nu+\mu}{2}+k-1} \\
& \times \theta\left(s_{1}-\frac{m_{1 Q}^{2}}{z(1-y)}-\frac{m_{2 Q}^{2}}{z y}\right)\left(\frac{d}{d s_{1}}\right)^{k} \delta\left(s_{2}-s_{1}\right) .
\end{aligned}
$$

If we look at the integrand out of the Step function $\theta$, it diverges inside the bound of $z$. However, this divergence is removed considering the step function coming from the successive application of the double Borel transformations by modifying the limits of the integral over $z$ and removing the points leading to the divergences out of the boundaries. Hence, we get finite results for the values of $n, k, \mu$, and $\nu$. Using this spectral density, the continuum subtracted correlation function in the Borel scheme corresponding to the considered term can be written as

$$
\Pi_{2}^{s u b}\left(M_{1}^{2}, M_{2}^{2}\right)=\int_{s_{L}}^{s_{0}} d s_{1} \int_{s_{L}}^{s_{0}} d s_{2} \rho\left(s_{1}, s_{2}\right) e^{-s_{1} / M_{1}^{2}} e^{-s_{2} / M_{2}^{2}}
$$

where $s_{L}=\left(m_{1 Q}+m_{2 Q}\right)^{2}$. Defining new variables, $s_{1}=2 s v$ and $s_{2}=2 s(1-v)$, we get

$$
\Pi_{2}^{s u b}\left(M_{1}^{2}, M_{2}^{2}\right)=\int_{s_{L}}^{s_{0}} d s \int_{0}^{1} d v \rho\left(s_{1}, s_{2}\right)(4 s) e^{-2 s v / M_{1}^{2}} e^{-2 s(1-v) / M_{2}^{2}} .
$$

Using the expression for the spectral density, one can get

$$
\begin{aligned}
\Pi_{2}^{s u b}\left(M_{1}^{2}, M_{2}^{2}\right) & =\mathcal{C} \sum_{k} a_{k} \frac{(-1)^{k}}{\Gamma\left(\frac{n+\nu+\mu}{2}+k\right)} \int_{s_{L}}^{s_{0}} d s \int_{0}^{1} d v \frac{1}{2^{k} s^{k}}\left(\left(\frac{d}{d v}\right)^{k} \delta(v-1 / 2)\right) \\
& \times \int_{0}^{2} d z \int_{0}^{1} d y z^{\nu+\mu-1}(1-z)^{\left(\frac{n-\nu-\mu}{2}\right)-1}(1-y)^{\nu-1} y^{\mu-1} \\
& \times\left(2 s v-\frac{m_{1 Q}^{2}}{z(1-y)}-\frac{m_{2 Q}^{2}}{z y}\right)^{\frac{n+\nu+\mu}{2}+k-1} e^{-2 s v / M_{1}^{2}} e^{-2 s(1-v) / M_{2}^{2}} \\
& \times \theta\left(2 s v-\frac{m_{1 Q}^{2}}{z(1-y)}-\frac{m_{2 Q}^{2}}{z y}\right) .
\end{aligned}
$$

Integrating over $v$, finally we obtain

$$
\begin{aligned}
\Pi_{2}^{s u b}\left(M_{1}^{2}, M_{2}^{2}\right) & =(-i) \frac{2^{2-n} \pi^{2}}{m_{1 Q}^{\nu} m_{2 Q}^{\mu} \Gamma\left(\frac{n-\nu-\mu}{2}\right)} \sum_{k} a_{k} \frac{1}{\Gamma\left(\frac{n+\nu+\mu}{2}+k\right)} \\
& \times \int_{s_{L}}^{s_{0}} d s \frac{1}{2^{k} s^{k}}\left[\left(\frac{d}{d v}\right)^{k} \int_{0}^{2} d z \theta\left(2 s v-\frac{m_{1 Q}^{2}}{z(1-y)}-\frac{m_{2 Q}^{2}}{z y}\right)\right. \\
& \times \int_{0}^{1} d y z^{\nu+\mu-1}(1-z)^{\left(\frac{n-\nu-\mu}{2}\right)-1}(1-y)^{\nu-1} y^{\mu-1} \\
& \left.\times\left(2 s v-\frac{m_{1 Q}^{2}}{z(1-y)}-\frac{m_{2 Q}^{2}}{z y}\right)^{\frac{n+\nu+\mu}{2}+k-1} e^{-2 s v / M_{1}^{2}} e^{-2 s(1-v) / M_{2}^{2}}\right]_{v=1 / 2}
\end{aligned}
$$




\subsection{Systems containing three heavy quarks}

In the following, we study hadrons containing triple heavy quarks with some light quarks. The general form of the correlation function in this case is

$$
T_{3}=\int_{0}^{1} d u \int d^{4} x e^{i P \cdot x} A(u) \frac{K_{\nu}\left(m_{1 Q} \sqrt{-x^{2}}\right) K_{\mu}\left(m_{2 Q} \sqrt{-x^{2}}\right) K_{\lambda}\left(m_{3 Q} \sqrt{-x^{2}}\right)}{\left(\sqrt{-x^{2}}\right)^{n}} .
$$

Using the definition of the modified Bessel function from Eq. (28), we obtain

$$
\begin{aligned}
T_{3} & =\frac{\Gamma(\nu+1 / 2) \Gamma(\mu+1 / 2) \Gamma(\lambda+1 / 2) 2^{\mu+\nu+\lambda}}{(\pi)^{3 / 2} m_{1 Q}^{\nu} m_{2 Q}^{\mu} m_{3 Q}^{\lambda}} \int_{0}^{1} d u \int d^{4} x e^{i P . x} A(u) \\
& \times \int_{0}^{\infty} d t_{3} \int_{0}^{\infty} d t_{2} \int_{0}^{\infty} d t_{1} \frac{\cos \left(m_{1 Q} t_{1}\right) \cos \left(m_{2 Q} t_{2}\right) \cos \left(m_{3 Q} t_{3}\right)}{\left(t_{1}^{2}-x^{2}\right)^{\nu+1 / 2}\left(t_{2}^{2}-x^{2}\right)^{\mu+1 / 2}\left(t_{3}^{2}-x^{2}\right)^{\lambda+1 / 2}\left(-x^{2}\right)^{\frac{n-\nu-\mu-\lambda}{2}}}
\end{aligned}
$$

where a Wick rotation and usage of the Schwinger integral representation leads to

$$
\begin{aligned}
T_{3} & =(-i) \frac{2^{\mu+\nu+\lambda}}{(\pi)^{3 / 2} m_{1 Q}^{\nu} m_{2 Q}^{\mu} m_{3 Q}^{\lambda} \Gamma\left(\frac{n-\nu-\mu-\lambda}{2}\right)} \int_{0}^{1} d u \int d^{4} x e^{-i P \cdot x} A(u) \int_{0}^{\infty} d t_{3} \int_{0}^{\infty} d t_{2} \\
& \times \int_{0}^{\infty} d t_{1} \int_{0}^{\infty} d y_{0} \int_{0}^{\infty} d y_{3} \int_{0}^{\infty} d y_{2} \int_{0}^{\infty} d y_{1} y_{1}^{\nu-\frac{1}{2}} e^{-y_{1}\left(x^{2}+t_{1}^{2}\right)} y_{2}^{\mu-\frac{1}{2}} e^{-y_{2}\left(x^{2}+t_{2}^{2}\right)} \\
& \times y_{3}^{\lambda-\frac{1}{2}} e^{-y_{3}\left(x^{2}+t_{3}^{2}\right)} y_{0}^{\frac{n-\nu-\mu-\lambda}{2}-1} e^{-y_{0} x^{2}} \cos \left(m_{1 Q} t_{1}\right) \cos \left(m_{2 Q} t_{2}\right) \cos \left(m_{3 Q} t_{3}\right) .
\end{aligned}
$$

Performing the $t_{i}$ 's and $x$-Gaussian integrals, we get

$$
\begin{aligned}
T_{3} & =(-i) \frac{2^{\mu+\nu+\lambda-3} \pi^{2}}{m_{1 Q}^{\nu} m_{2 Q}^{\mu} m_{3 Q}^{\lambda} \Gamma\left(\frac{n-\nu-\mu-\lambda}{2}\right)} \int_{0}^{1} d u A(u) \int_{0}^{\infty} d y_{0} y_{0}^{\frac{n-\nu-\mu-\lambda}{2}-1} \\
& \times \int_{0}^{\infty} d y_{3} \int_{0}^{\infty} d y_{2} \int_{0}^{\infty} d y_{1} \frac{y_{1}^{\nu-1} e^{-\frac{m_{1 Q}^{2}}{4 y_{1}}} y_{2}^{\mu-1} e^{-\frac{m_{2 Q}^{2}}{4 y_{2}}} y_{3}^{\lambda-1} e^{-\frac{m_{3 Q}^{2}}{4 y_{3}}} e^{\frac{-P^{2}}{4\left(y_{0}+y_{1}+y_{2}+y_{3}\right)}}}{\left(y_{0}+y_{1}+y_{2}+y_{3}\right)^{2}}
\end{aligned}
$$

We introduce the variables $\rho, v, r$ and $w$ defined by

$$
\begin{aligned}
\rho & =y_{0}+y_{1}+y_{2}+y_{3}, \quad v=\frac{y_{1}}{y_{0}+y_{1}+y_{2}+y_{3}}, \quad r=\frac{y_{2}}{y_{0}+y_{1}+y_{2}+y_{3}}, \\
w & =\frac{y_{3}}{y_{0}+y_{1}+y_{2}+y_{3}},
\end{aligned}
$$

which leads to

$$
\begin{aligned}
T_{3} & =(-i) \frac{2^{\mu+\nu+\lambda-3} \pi^{2}}{m_{1 Q}^{\nu} m_{2 Q}^{\mu} m_{3 Q}^{\lambda} \Gamma\left(\frac{n-\nu-\mu-\lambda}{2}\right)} \int_{0}^{1} d u A(u) \int_{0}^{1} d w(\rho(1-v-r-w))^{\frac{n-\nu-\mu-\lambda}{2}-1} \\
& \times \int_{0}^{\infty} \rho d \rho \int_{0}^{1} d v \int_{0}^{1} d r(\rho v)^{\nu-1} e^{-\frac{m_{1 Q}^{2}}{4 \rho v}}(\rho r)^{\mu-1} e^{-\frac{m_{2 Q}^{2}}{4 \rho r}}(\rho w)^{\lambda-1} e^{-\frac{m_{3 Q}^{2}}{4 \rho w}} e^{\frac{-P^{2}}{4 \rho}}
\end{aligned}
$$

Now we apply the Borel transformation of the exponential $e^{\frac{-P^{2}}{4 \rho}}$, and perform the $u$ and 
$\rho$ integrals. As a result we get

$$
\begin{aligned}
& \mathcal{B}_{M_{1}} \mathcal{B}_{M_{2}} T_{3}=(-i) \frac{2^{1-n} \pi^{2}}{m_{1 Q}^{\nu} m_{2 Q}^{\mu} m_{3 Q}^{\lambda} \Gamma\left(\frac{n-\nu-\mu-\lambda}{2}\right)} A\left(u_{0}\right)\left(M^{2}\right)^{\frac{n+\nu+\mu+\lambda}{2}} e^{\frac{q^{2}}{M_{1}^{2}+M_{2}^{2}}} \int_{0}^{1} d w \int_{0}^{1} d v \\
& \times \quad \int_{0}^{1} d r(1-v-r-w)^{\frac{n-\nu-\mu-\lambda}{2}-1} v^{\nu-1} e^{-\frac{m_{1 Q}^{2}}{M^{2} v}} r^{\mu-1} e^{-\frac{m_{2 Q}^{2}}{M^{2} r}} w^{\lambda-1} e^{-\frac{m_{3 Q}^{3}}{M^{2} w}} .
\end{aligned}
$$

The variables $x, y$ and $z$, defined by

$$
v=z x(1-y), \quad r=z x y, \quad w=z(1-x),
$$

help us end up in the final form of the double Borel transformed $T$ function as

$$
\begin{aligned}
\mathcal{B}_{M_{1}} \mathcal{B}_{M_{2}} T_{3} & =(-i) \frac{2^{1-n} \pi^{2}}{m_{1 Q}^{\nu} m_{2 Q}^{\mu} m_{3 Q}^{\lambda} \Gamma\left(\frac{n-\nu-\mu-\lambda}{2}\right)} A\left(u_{0}\right)\left(M^{2}\right)^{\frac{n+\nu+\mu+\lambda}{2}} e^{\frac{q^{2}}{M_{1}^{2}+M_{2}^{2}}} \int_{0}^{1} d x \int_{0}^{1} d y \\
& \times \int_{0}^{3} d z(1-z)^{\frac{n-\nu-\mu-\lambda}{2}-1} z^{\nu+\mu+\lambda-1} x^{\nu+\mu-1}(1-x)^{\lambda-1}(1-y)^{\nu-1} y^{\mu-1} \\
& \times e^{-\frac{m_{1 Q}^{2}}{M^{2} z x(1-y)}} e^{-\frac{m_{2 Q}^{2}}{M^{2} z x y}} e^{-\frac{m_{3 Q}^{2}}{M^{2} z(1-x)}}
\end{aligned}
$$

\section{Spectral representation and continuum subtraction for systems containing three heavy quarks}

To suppress the contribution of the higher states and continuum in this case we consider a general form as fallows

$$
\begin{aligned}
\Pi_{3} & =\mathcal{C}\left(M^{2}\right)^{\frac{n+\nu+\mu+\lambda}{2}} A\left(u_{0}\right) \int_{0}^{1} d x \int_{0}^{1} d y \int_{0}^{3} d z(1-z)^{\frac{n-\nu-\mu-\lambda}{2}-1} z^{\nu+\mu+\lambda-1} \\
& \times \quad x^{\nu+\mu-1}(1-x)^{\lambda-1}(1-y)^{\nu-1} y^{\mu-1} e^{-\frac{m_{1 Q}^{2}}{M^{2} z x(1-y)}} e^{-\frac{m_{2 Q}^{2}}{M^{2} z x y}} e^{-\frac{m_{3 Q}^{2}}{M^{2} z(1-x)}} .
\end{aligned}
$$

where

$$
\mathcal{C}=(-i) \frac{2^{1-n} \pi^{2}}{m_{1 Q}^{\nu} m_{2 Q}^{\mu} m_{3 Q}^{\lambda} \Gamma\left(\frac{n-\nu-\mu-\lambda}{2}\right)}
$$

Expressing $A\left(u_{0}\right)$ function in series, leads to

$$
\begin{aligned}
\Pi_{3} & =\mathcal{C}\left(\frac{M_{1}^{2} M_{2}^{2}}{M_{1}^{2}+M_{2}^{2}}\right)^{\frac{n+\nu+\mu+\lambda}{2}} \sum_{k} a_{k}\left(\frac{M_{2}^{2}}{M_{1}^{2}+M_{2}^{2}}\right)^{k} \int_{0}^{1} d x \int_{0}^{1} d y \int_{0}^{3} d z(1-z)^{\frac{n-\nu-\mu-\lambda}{2}-1} \\
& \times z^{\nu+\mu+\lambda-1} x^{\nu+\mu-1}(1-x)^{\lambda-1}(1-y)^{\nu-1} y^{\mu-1} e^{-\frac{m_{1 Q}^{2}}{M^{2} z x(1-y)}} e^{-\frac{m_{2 Q}^{2}}{M^{2} z x y}} e^{-\frac{m_{3 Q}^{2}}{M^{2} z(1-x)}}
\end{aligned}
$$


We introduce the new variables, $\sigma_{i}=\frac{1}{M_{i}^{2}}$, and rearrange the terms

$$
\begin{aligned}
& \Pi_{3}=\mathcal{C} \sum_{k} a_{k} \frac{\sigma_{1}^{k}}{\left(\sigma_{1}+\sigma_{2}\right)^{\frac{n+\nu+\mu+\lambda}{2}+k}} \int_{0}^{1} d x \int_{0}^{1} d y \int_{0}^{3} d z(1-z)^{\frac{n-\nu-\mu-\lambda}{2}-1} z^{\nu+\mu+\lambda-1} \\
& \times \quad x^{\nu+\mu-1}(1-x)^{\lambda-1}(1-y)^{\nu-1} y^{\mu-1} e^{-\frac{m_{1 Q}^{2}\left(\sigma_{1}+\sigma_{2}\right)}{z x(1-y)}} e^{-\frac{m_{2 Q}^{2}\left(\sigma_{1}+\sigma_{2}\right)}{z x y}} e^{-\frac{m_{3 Q}^{2}\left(\sigma_{1}+\sigma_{2}\right)}{z(1-x)}} \\
& =\mathcal{C} \sum_{k} a_{k} \frac{\sigma_{1}^{k}}{\Gamma\left(\frac{n+\nu+\mu+\lambda}{2}+k\right)} \int_{0}^{1} d x \int_{0}^{1} d y \int_{0}^{3} d z(1-z)^{\frac{n-\nu-\mu-\lambda}{2}-1} z^{\nu+\mu+\lambda-1} \\
& \times \quad x^{\nu+\mu-1}(1-x)^{\lambda-1}(1-y)^{\nu-1} y^{\mu-1} e^{-\frac{m_{1 Q}^{2}\left(\sigma_{1}+\sigma_{2}\right)}{z x(1-y)}} e^{-\frac{m_{2 Q}^{2}\left(\sigma_{1}+\sigma_{2}\right)}{z x y}} e^{-\frac{m_{3 Q}^{2}\left(\sigma_{1}+\sigma_{2}\right)}{z(1-x)}} \\
& \times \int_{0}^{\infty} d l e^{-l\left(\sigma_{1}+\sigma_{2}\right)} l^{\frac{n+\nu+\mu+\lambda}{2}+k-1} \\
& =\mathcal{C} \sum_{k} a_{k} \frac{\sigma_{1}^{k}}{\Gamma\left(\frac{n+\nu+\mu+\lambda}{2}+k\right)} \int_{0}^{1} d x \int_{0}^{1} d y \int_{0}^{3} d z(1-z)^{\frac{n-\nu-\mu-\lambda}{2}-1} z^{\nu+\mu+\lambda-1} x^{\nu+\mu-1}(1-x)^{\lambda-1} \\
& \times \quad \int_{0}^{\infty} d l l^{\frac{n+\nu+\mu+\lambda}{2}+k-1} e^{-\left(l+\frac{m_{1 Q}^{2}}{z x(1-y)}+\frac{m_{2 Q}^{2}}{z x y}+\frac{m_{3 Q}^{2}}{z(1-x)}\right)\left(\sigma_{1}+\sigma_{2}\right)}(1-y)^{\nu-1} y^{\mu-1} \\
& =\mathcal{C} \sum_{k} a_{k} \frac{(-1)^{k}}{\Gamma\left(\frac{n+\nu+\mu+\lambda}{2}+k\right)} \int_{0}^{1} d x \int_{0}^{1} d y \int_{0}^{3} d z(1-z)^{\frac{n-\nu-\mu-\lambda}{2}-1} z^{\nu+\mu+\lambda-1} x^{\nu+\mu-1}(1-x)^{\lambda-1} \\
& \times \quad \int_{0}^{\infty} d l l^{\frac{n+\nu+\mu+\lambda}{2}+k-1}\left(\left(\frac{d}{d l}\right)^{k} e^{-\left(l+\frac{m_{1 Q}^{2}}{z x(1-y)}+\frac{m_{2 Q}^{2}}{z x y}+\frac{m_{3 Q}^{2}}{z(1-x)}\right) \sigma_{1}}\right) \\
& \times \quad e^{-\left(l+\frac{m_{1 Q}^{2}}{z x(1-y)}+\frac{m_{2 Q}^{2}}{z x y}+\frac{m_{3 Q}^{2}}{z(1-x)}\right) \sigma_{2}}(1-y)^{\nu-1} y^{\mu-1} .
\end{aligned}
$$

Applying double Borel transformation with respect to $\sigma_{1} \rightarrow \frac{1}{\tau_{1}}$ and $\sigma_{2} \rightarrow \frac{1}{\tau_{2}}$, gives us

$$
\begin{aligned}
\mathcal{B}_{1 / \tau_{1}} \mathcal{B}_{1 / \tau_{2}} \Pi_{3} & =\mathcal{C} \sum_{k} a_{k} \frac{(-1)^{k}}{\Gamma\left(\frac{n+\nu+\mu+\lambda}{2}+k\right)} \int_{0}^{1} d x \int_{0}^{1} d y \int_{0}^{3} d z(1-z)^{\frac{n-\nu-\mu-\lambda}{2}-1} z^{\nu+\mu+\lambda-1} \\
& \times x^{\nu+\mu-1}(1-x)^{\lambda-1}(1-y)^{\nu-1} y^{\mu-1} \int_{0}^{\infty} d l l^{\frac{n+\nu+\mu+\lambda}{2}+k-1} \\
& \times\left(\left(\frac{d}{d l}\right)^{k} \delta\left(\tau_{1}-\left(l+\frac{m_{1 Q}^{2}}{z x(1-y)}+\frac{m_{2 Q}^{2}}{z x y}+\frac{m_{3 Q}^{2}}{z(1-x)}\right)\right)\right. \\
& \times \delta\left(\tau_{2}-\left(l+\frac{m_{1 Q}^{2}}{z x(1-y)}+\frac{m_{2 Q}^{2}}{z x y}+\frac{m_{3 Q}^{2}}{z(1-x)}\right)\right)
\end{aligned}
$$

where the spectral density $\rho_{3}\left(s_{1}, s_{2}\right)$ is found from the same manner as the previous sections as

$$
\begin{aligned}
\rho_{3}\left(s_{1}, s_{2}\right) & =\mathcal{C} \sum_{k} a_{k} \frac{(-1)^{k}}{\Gamma\left(\frac{n+\nu+\mu+\lambda}{2}+k\right)} \int_{0}^{1} d x \int_{0}^{1} d y \int_{0}^{3} d z(1-z)^{\frac{n-\nu-\mu-\lambda}{2}-1} z^{\nu+\mu+\lambda-1} \\
& \times x^{\nu+\mu-1}(1-x)^{\lambda-1}(1-y)^{\nu-1} y^{\mu-1} \int_{0}^{\infty} d l l^{\frac{n+\nu+\mu+\lambda}{2}+k-1} \\
& \times\left(\left(\frac{d}{d l}\right)^{k} \delta\left(s_{1}-\left(l+\frac{m_{1 Q}^{2}}{z x(1-y)}+\frac{m_{2 Q}^{2}}{z x y}+\frac{m_{3 Q}^{2}}{z(1-x)}\right)\right)\right. \\
& \times \delta\left(s_{2}-\left(l+\frac{m_{1 Q}^{2}}{z x(1-y)}+\frac{m_{2 Q}^{2}}{z x y}+\frac{m_{3 Q}^{2}}{z(1-x)}\right)\right) .
\end{aligned}
$$


Performing integration over $l$, finally we obtain the following expression for the double spectral density:

$$
\begin{aligned}
\rho_{3}\left(s_{1}, s_{2}\right) & =\mathcal{C} \sum_{k} a_{k} \frac{(-1)^{k}}{\Gamma\left(\frac{n+\nu+\mu+\lambda}{2}+k\right)} \int_{0}^{1} d x \int_{0}^{1} d y \int_{0}^{3} d z(1-z)^{\frac{n-\nu-\mu-\lambda}{2}-1} z^{\nu+\mu+\lambda-1} x^{\nu+\mu-1} \\
& \times(1-x)^{\lambda-1}(1-y)^{\nu-1} y^{\mu-1}\left(s_{1}-\frac{m_{1 Q}^{2}}{z x(1-y)}-\frac{m_{2 Q}^{2}}{z x y}-\frac{m_{3 Q}^{2}}{z(1-x)}\right)^{\frac{n+\nu+\mu+\lambda}{2}+k-1} \\
& \times\left(\left(\frac{d}{d s_{1}}\right)^{k} \delta\left(s_{2}-s_{1}\right)\right) \theta\left(s_{1}-\frac{m_{1 Q}^{2}}{z x(1-y)}-\frac{m_{2 Q}^{2}}{z x y}-\frac{m_{3 Q}^{2}}{z(1-x)}\right)
\end{aligned}
$$

Using this spectral density, the continuum subtracted correlation function in the Borel scheme corresponding to the considered term can be written as:

$$
\Pi_{3}^{s u b}\left(M_{1}^{2}, M_{2}^{2}\right)=\int_{s_{L}}^{s_{0}} d s_{1} \int_{s_{L}}^{s_{0}} d s_{2} \rho\left(s_{1}, s_{2}\right) e^{-s_{1} / M_{1}^{2}} e^{-s_{2} / M_{2}^{2}},
$$

where $s_{L}=\left(m_{1 Q}+m_{2 Q}+m_{3 Q}\right)^{2}$. Defining new variables, $s_{1}=2 s v$ and $s_{2}=2 s(1-v)$, we get

$$
\Pi_{3}^{s u b}\left(M_{1}^{2}, M_{2}^{2}\right)=\int_{s_{L}}^{s_{0}} d s \int_{0}^{1} d v \rho\left(s_{1}, s_{2}\right)(4 s) e^{-2 s v / M_{1}^{2}} e^{-2 s(1-v) / M_{2}^{2}} .
$$

Using the expression for the spectral density, one can get

$$
\begin{aligned}
\Pi_{3}^{s u b}\left(M_{1}^{2}, M_{2}^{2}\right) & =\mathcal{C} \sum_{k} a_{k} \frac{(-1)^{k}}{\Gamma\left(\frac{n+\nu+\mu+\lambda}{2}+k\right)} \int_{s_{L}}^{s_{0}} d s \int_{0}^{1} d v \frac{1}{2^{k} s^{k}}\left(\left(\frac{d}{d v}\right)^{k} \delta(v-1 / 2)\right) \\
& \times \int_{0}^{1} d x \int_{0}^{1} d y \int_{0}^{3} d z(1-z)^{\frac{n-\nu-\mu-\lambda}{2}-1} z^{\nu+\mu+\lambda-1} x^{\nu+\mu-1}(1-x)^{\lambda-1} \\
& \times(1-y)^{\nu-1} y^{\mu-1}\left(2 s v-\frac{m_{1 Q}^{2}}{z x(1-y)}-\frac{m_{2 Q}^{2}}{z x y}-\frac{m_{3 Q}^{2}}{z(1-x)}\right)^{\frac{n+\nu+\mu+\lambda}{2}+k-1} \\
& \times \theta\left(2 s v-\frac{m_{1 Q}^{2}}{z x(1-y)}-\frac{m_{2 Q}^{2}}{z x y}-\frac{m_{3 Q}^{2}}{z(1-x)}\right) e^{-2 s v / M_{1}^{2}} e^{-2 s(1-v) / M_{2}^{2}}
\end{aligned}
$$

Integrating over $v$, finally we obtain

$$
\begin{aligned}
\Pi_{3}^{s u b}\left(M_{1}^{2}, M_{2}^{2}\right) & =(-i) \sum_{k} a_{k} \frac{2^{1-n} \pi^{2}}{m_{1 Q}^{\nu} m_{2 Q}^{\mu} m_{3 Q}^{\lambda} \Gamma\left(\frac{n-\nu-\mu-\lambda}{2}\right) \Gamma\left(\frac{n+\nu+\mu+\lambda}{2}+k\right)} \\
& \times \int d s \frac{1}{2^{k} s^{k}}\left[\left(\frac{d}{d v}\right)^{k} e^{-2 s v / M_{1}^{2}} e^{-2 s(1-v) / M_{2}^{2}}\right. \\
& \times \int_{0}^{3} d z \theta\left(2 s v-\frac{m_{1 Q}^{2}}{z x(1-y)}-\frac{m_{2 Q}^{2}}{z x y}-\frac{m_{3 Q}^{2}}{z(1-x)}\right) \\
& \times \int_{0}^{1} d x \int_{0}^{1} d y(1-z)^{\frac{n-\nu-\mu-\lambda}{2}}-1 z^{\nu+\mu+\lambda-1} x^{\nu+\mu-1}(1-x)^{\lambda-1}(1-y)^{\nu-1} y^{\mu-1} \\
& \left.\times\left(2 s v-\frac{m_{1 Q}^{2}}{z x(1-y)}-\frac{m_{2 Q}^{2}}{z x y}-\frac{m_{3 Q}^{2}}{z(1-x)}\right)^{\frac{n+\nu+\mu+\lambda}{2}+k-1}\right]_{v=1 / 2}
\end{aligned}
$$




\subsection{Systems containing four heavy quarks}

In the following, we study hadrons containing four heavy and some light quarks. We start with the generic term:

$T_{4}=\int_{0}^{1} d u \int d^{4} x e^{i P . x} A(u) \frac{K_{\nu}\left(m_{1 Q} \sqrt{-x^{2}}\right) K_{\mu}\left(m_{2 Q} \sqrt{-x^{2}}\right) K_{\lambda}\left(m_{3 Q} \sqrt{-x^{2}}\right) K_{\eta}\left(m_{4 Q} \sqrt{-x^{2}}\right)}{\left(\sqrt{-x^{2}}\right)^{n}}$,

where $m_{i Q}$ are again the masses of the heavy quarks. We can rewrite Eq. (98) as

$$
\begin{aligned}
T_{4} & =\frac{\Gamma(\nu+1 / 2) \Gamma(\mu+1 / 2) \Gamma(\lambda+1 / 2) \Gamma(\eta+1 / 2) 2^{\mu+\nu+\lambda+\eta}}{(\pi)^{2} m_{1 Q}^{\nu} m_{2 Q}^{\mu} m_{3 Q}^{\lambda} m_{4 Q}^{\eta}} \\
& \times \int_{0}^{1} d u \int d^{4} x e^{i P . x} A(u) \frac{1}{\left(-x^{2}\right)^{\frac{n-\mu-\nu-\lambda-\eta}{2}}} \int_{0}^{\infty} d t_{4} \int_{0}^{\infty} d t_{3} \int_{0}^{\infty} d t_{2} \\
& \times \int_{0}^{\infty} d t_{1} \frac{\cos \left(m_{1 Q} t_{1}\right) \cos \left(m_{2 Q} t_{2}\right) \cos \left(m_{3 Q} t_{3}\right) \cos \left(m_{4 Q} t_{4}\right)}{\left(t_{1}^{2}-x^{2}\right)^{\nu+1 / 2}\left(t_{2}^{2}-x^{2}\right)^{\mu+1 / 2}\left(t_{3}^{2}-x^{2}\right)^{\lambda+1 / 2}\left(t_{4}^{2}-x^{2}\right)^{\eta+1 / 2}} .
\end{aligned}
$$

We perform a Wick rotation to go to the Euclidean space and use again the Schwinger integral representation, we get

$$
\begin{aligned}
T_{4} & =\frac{(-i) 2^{\mu+\nu+\lambda+\eta}}{(\pi)^{2} m_{1 Q}^{\nu} m_{2 Q}^{\mu} m_{3 Q}^{\lambda} m_{4 Q}^{\eta} \Gamma\left(\frac{n-\nu-\mu-\lambda-\eta}{2}\right)} \int_{0}^{1} d u \int d^{4} x e^{-i P \cdot x} A(u) \int_{0}^{\infty} d t_{4} \int_{0}^{\infty} d t_{3} \int_{0}^{\infty} d t_{2} \\
& \times \int_{0}^{\infty} d t_{1} \int_{0}^{\infty} d y_{4} \int_{0}^{\infty} d y_{3} \int_{0}^{\infty} d y_{2} \int_{0}^{\infty} d y_{1} \int_{0}^{\infty} d y_{0} y_{0}^{\frac{n-\nu-\mu-\lambda}{2}-1} e^{-y_{0} x^{2}} \\
& \times y_{1}^{\nu-\frac{1}{2}} e^{-y_{1}\left(x^{2}+t_{1}^{2}\right)} y_{2}^{\mu-\frac{1}{2}} e^{-y_{2}\left(x^{2}+t_{2}^{2}\right)} y_{3}^{\lambda-\frac{1}{2}} e^{-y_{3}\left(x^{2}+t_{3}^{2}\right)} y_{4}^{\eta-\frac{1}{2}} e^{-y_{4}\left(x^{2}+t_{4}^{2}\right)} \\
& \times \cos \left(m_{1 Q} t_{1}\right) \cos \left(m_{2 Q} t_{2}\right) \cos \left(m_{3 Q} t_{3}\right) \cos \left(m_{4 Q} t_{4}\right) .
\end{aligned}
$$

The next step is to perform the Gaussian integral over four- $x$ and integrals over parameters $t_{i}$. As a result, we get we get

$$
\begin{aligned}
T_{4} & =(-i) \frac{2^{\mu+\nu+\lambda+\eta-4} \pi^{2}}{m_{1 Q}^{\nu} m_{2 Q}^{\mu} m_{3 Q}^{\lambda} m_{4 Q}^{\eta} \Gamma\left(\frac{n-\nu-\mu-\lambda-\eta}{2}\right)} \int_{0}^{1} d u A(u) \int_{0}^{\infty} d y_{0} y_{0}^{\frac{n-\nu-\mu-\lambda-\eta}{2}-1} e^{\frac{-P^{2}}{\left(y_{0}+y_{1}+y_{2}+y_{3}+y_{4}\right)}} \\
& \times \int_{0}^{\infty} d y_{4} \int_{0}^{\infty} d y_{3} \int_{0}^{\infty} d y_{2} \int_{0}^{\infty} d y_{1} \frac{y_{1}^{\nu-1} e^{-\frac{m_{1 Q}^{2}}{4 y_{1}}} y_{2}^{\mu-1} e^{-\frac{m_{2 Q}^{2}}{4 y_{2}}} y_{3}^{\lambda-1} e^{-\frac{m_{3 Q}^{2}}{4 y_{3}}} y_{4}^{\eta-1} e^{-\frac{m_{4 Q}^{2}}{4 y_{4}}}}{\left(y_{0}+y_{1}+y_{2}+y_{3}+y_{4}\right)^{2}} .
\end{aligned}
$$

We introduce the new set of variable as

$$
\begin{array}{ll}
\rho=y_{0}+y_{1}+y_{2}+y_{3}+y_{4}, & v=\frac{y_{1}}{y_{0}+y_{1}+y_{2}+y_{3}+y_{4}}, \\
r=\frac{y_{2}}{y_{0}+y_{1}+y_{2}+y_{3}+y_{4}}, & w=\frac{y_{4}}{y_{0}+y_{1}+y_{2}+y_{3}+y_{4}}, \\
l=\frac{y_{0}+y_{1}+y_{2}+y_{3}+y_{4}}{y_{0}}, &
\end{array}
$$


which leads to

$$
\begin{aligned}
& T_{4}=(-i) \frac{2^{\mu+\nu+\lambda+\eta-4} \pi^{2}}{m_{1 Q}^{\nu} m_{2 Q}^{\mu} m_{3 Q}^{\lambda} m_{4 Q}^{\eta} \Gamma\left(\frac{n-\nu-\mu-\lambda-\eta}{2}\right)} \int_{0}^{1} d u A(u) \\
& \times \quad \int_{0}^{1} d w(\rho(1-v-r-l-w))^{\frac{n-\nu-\mu-\lambda-\eta}{2}-1} \int_{0}^{\infty} \rho^{2} d \rho \int_{0}^{1} d v \int_{0}^{1} d r \\
& \times \quad \int_{0}^{1} d l(\rho v)^{\nu-1} e^{-\frac{m_{1 Q}^{2}}{4 \rho v}}(\rho r)^{\mu-1} e^{-\frac{m_{2 Q}^{2}}{4 \rho r}}(\rho w)^{\lambda-1} e^{-\frac{m_{3 Q}^{2}}{4 \rho w}}(\rho l)^{\eta-1} e^{-\frac{m_{4 Q}^{2}}{4 \rho l}} e^{\frac{-P^{2}}{4 \rho}} .
\end{aligned}
$$

Applying the double Borel transformation and performing integral over the parameters $u$ and $\rho$, we obtain

$$
\begin{aligned}
\mathcal{B}_{M_{1}} \mathcal{B}_{M_{2}} T_{4} & =(-i) \frac{2^{-n} \pi^{2}}{m_{1 Q}^{\nu} m_{2 Q}^{\mu} m_{3 Q}^{\lambda} m_{4 Q}^{\eta} \Gamma\left(\frac{n-\nu-\mu-\lambda-\eta}{2}\right)} A\left(u_{0}\right)\left(M^{2}\right)^{\frac{n+\mu+\nu+\lambda+\eta}{2}} e^{\frac{q^{2}}{M_{1}^{2}+M_{2}^{2}}} \\
& \times \int_{0}^{1} d v \int_{0}^{1} d r \int_{0}^{1} d l \int_{0}^{1} d w(1-v-r-l-w)^{\frac{n-\nu-\mu-\lambda-\eta}{2}-1} \\
& \times v^{\nu-1} e^{-\frac{m_{1 Q}^{2}}{M^{2} v}} r^{\mu-1} e^{-\frac{m_{2 Q}^{2}}{M^{2} r}} w^{\lambda-1} e^{-\frac{m_{3 Q}^{2}}{M^{2} w}} l^{\eta-1} e^{-\frac{m_{4 Q}^{2}}{M^{2} l}}
\end{aligned}
$$

For further simplifications, we introduce the variables $x, y, z$ and $t$, defined by

$$
v=z x t(1-y), \quad r=z x y t, \quad w=z t(1-x), \quad l=z(1-t) .
$$

Hence,

$$
\begin{aligned}
\mathcal{B}_{M_{1}} \mathcal{B}_{M_{2}} T_{4} & =(-i) \frac{2^{-n} \pi^{2}}{m_{1 Q}^{\nu} m_{2 Q}^{\mu} m_{3 Q}^{\lambda} m_{4 Q}^{\eta} \Gamma\left(\frac{n-\nu-\mu-\lambda-\eta}{2}\right)} A\left(u_{0}\right)\left(M^{2}\right)^{\frac{n+\mu+\nu+\lambda+\eta}{2}} e^{\frac{q^{2}}{M_{1}^{2}+M_{2}^{2}}} \\
& \times \int_{0}^{4} d z \int_{0}^{1} d x \int_{0}^{1} d y \int_{0}^{1} d t z^{\nu+\mu+\lambda+\eta-1}(1-z)^{\frac{n-\nu-\mu-\lambda-\eta}{2}-1} t^{\nu+\mu+\lambda-1}(1-t)^{\eta-1} \\
& \times x^{\nu+\mu-1}(1-x)^{\lambda-1} y^{\mu-1}(1-y)^{\nu-1} e^{-\frac{m_{1 Q}^{2}}{M^{2} z x t(1-y)}} e^{-\frac{m_{2 Q}^{2}}{M^{2} z x y t}} e^{-\frac{m_{3 Q}^{2}}{M^{2} z t(1-x)}} e^{-\frac{m_{4 Q}^{2}}{M^{2} z(1-t)}}
\end{aligned}
$$

\section{Spectral representation and continuum subtraction for systems containing four heavy quarks}

We again start with the fallowing general form:

$$
\begin{aligned}
\Pi_{4} & =\mathcal{C}\left(M^{2}\right)^{\frac{n+\mu+\nu+\lambda+\eta}{2}} A\left(u_{0}\right) \int_{0}^{4} d z \int_{0}^{1} d x \int_{0}^{1} d y \int_{0}^{1} d t z^{\nu+\mu+\lambda+\eta-1}(1-z)^{\frac{n-\nu-\mu-\lambda-\eta}{2}-1} \\
& \times t^{\nu+\mu+\lambda-1}(1-t)^{\eta-1} x^{\nu+\mu-1}(1-x)^{\lambda-1} y^{\mu-1}(1-y)^{\nu-1} e^{-\frac{m_{1 Q}^{2}}{M^{2} z x t(1-y)}} \\
& \times e^{-\frac{m_{2 Q}^{2}}{M^{2} z x y t}} e^{-\frac{m_{3 Q}^{2}}{M^{2} z t(1-x)}} e^{-\frac{m_{4 Q}^{2}}{M^{2} z(1-t)}}
\end{aligned}
$$

where

$$
\mathcal{C}=(-i) \frac{2^{-n} \pi^{2}}{m_{1 Q}^{\nu} m_{2 Q}^{\mu} m_{3 Q}^{\lambda} m_{4 Q}^{\eta} \Gamma\left(\frac{n-\nu-\mu-\lambda-\eta}{2}\right)} .
$$


As usual, the first step is to expand $A\left(u_{0}\right)$ in series, which leads to

$$
\begin{aligned}
\Pi_{4} & =\mathcal{C}\left(\frac{M_{1}^{2} M_{2}^{2}}{M_{1}^{2}+M_{2}^{2}}\right)^{\frac{n+\mu+\nu+\lambda+\eta}{2}} \sum a_{k}\left(\frac{M_{2}^{2}}{M_{1}^{2}+M_{2}^{2}}\right)^{k} \int_{0}^{4} d z \int_{0}^{1} d x \int_{0}^{1} d y \int_{0}^{1} d t z^{\nu+\mu+\lambda+\eta-1} \\
& \times(1-z)^{\frac{n-\nu-\mu-\lambda-\eta}{2}-1} t^{\nu+\mu+\lambda-1}(1-t)^{\eta-1} x^{\nu+\mu-1}(1-x)^{\lambda-1} \\
& \times y^{\mu-1}(1-y)^{\nu-1} e^{-\frac{m_{1 Q}^{2}}{M^{2} z x t(1-y)}} e^{-\frac{m_{2 Q}^{2}}{M^{2} z x y t}} e^{-\frac{m_{3 Q}^{2}}{M^{2} z t(1-x)}} e^{-\frac{m_{4 Q}^{2}}{M^{2} z(1-t)}}
\end{aligned}
$$

Now, the new variables, $\sigma_{i}=\frac{1}{M_{i}^{2}}$, are introduced. After some manipulations, we get

$$
\begin{aligned}
\Pi_{4} & =\mathcal{C} \sum_{k} a_{k} \frac{(-1)^{k}}{\Gamma\left(\frac{n+\mu+\nu+\lambda+\eta}{2}+k\right)} \int_{0}^{4} d z \int_{0}^{1} d x \int_{0}^{1} d y \int_{0}^{1} d t z^{\nu+\mu+\lambda+\eta-1}(1-z)^{\frac{n-\nu-\mu-\lambda-\eta}{2}-1} \\
& \times y^{\mu-1}(1-y)^{\nu-1} t^{\nu+\mu+\lambda-1}(1-t)^{\eta-1} x^{\nu+\mu-1}(1-x)^{\lambda-1} \\
& \times \int_{0}^{\infty} d l l^{\frac{n+\mu+\nu+\lambda+\eta}{2}+k-1}\left(\left(\frac{d}{d l}\right)^{k} e^{-\left(l+\frac{m_{1 Q}^{2}}{z x t(1-y)}+\frac{m_{2 Q}^{2}}{z x y t}+\frac{m_{3 Q}^{2}}{z t(1-x)}+\frac{m_{4 Q}^{2}}{z(1-t)}\right) \sigma_{1}}\right) \\
& \times \quad e^{-\left(l+\frac{m_{1 Q}^{2}}{z x t(1-y)}+\frac{m_{2 Q}^{2}}{z x y t}+\frac{m_{3 Q}^{2}}{z t(1-x)}+\frac{m_{4 Q}^{2}}{z(1-t)}\right) \sigma_{2}} .
\end{aligned}
$$

The double Borel transformation with respect to $\sigma_{1} \rightarrow \frac{1}{s_{1}}$ and $\sigma_{2} \rightarrow \frac{1}{s_{2}}$ are applied. From similar manner to the previous cases, the spectral density is found as

$$
\begin{aligned}
\rho_{4}\left(s_{1}, s_{2}\right) & =\mathcal{C} \sum_{k} a_{k} \frac{(-1)^{k}}{\Gamma\left(\frac{n+\mu+\nu+\lambda+\eta}{2}+k\right)} \int_{0}^{4} d z \int_{0}^{1} d x \int_{0}^{1} d y \int_{0}^{1} d t z^{\nu+\mu+\lambda+\eta-1}(1-z)^{\frac{n-\nu-\mu-\lambda-\eta}{2}-1} \\
& \times y^{\mu-1}(1-y)^{\nu-1} t^{\nu+\mu+\lambda-1}(1-t)^{\eta-1} x^{\nu+\mu-1}(1-x)^{\lambda-1} \\
& \times \int_{0}^{\infty} d l l^{\frac{n+\mu+\nu+\lambda+\eta}{2}+k-1}\left(\left(\frac{d}{d l}\right)^{k} \delta\left(s_{1}-\left(l+\frac{m_{1 Q}^{2}}{z x t(1-y)}+\frac{m_{2 Q}^{2}}{z x y t}+\frac{m_{3 Q}^{2}}{z t(1-x)}+\frac{m_{4 Q}^{2}}{z(1-t)}\right)\right)\right. \\
& \times \delta\left(s_{2}-\left(l+\frac{m_{1 Q}^{2}}{z x t(1-y)}+\frac{m_{2 Q}^{2}}{z x y t}+\frac{m_{3 Q}^{2}}{z t(1-x)}+\frac{m_{4 Q}^{2}}{z(1-t)}\right)\right) .
\end{aligned}
$$

Performing the integration over $l$, finally we obtain the following expression for the double spectral density:

$$
\begin{aligned}
\rho_{4}\left(s_{1}, s_{2}\right) & =\mathcal{C} \sum_{k} a_{k} \frac{(-1)^{k}}{\Gamma\left(\frac{n+\mu+\nu+\lambda+\eta}{2}+k\right)} \int_{0}^{4} d z \int_{0}^{1} d x \int_{0}^{1} d y \int_{0}^{1} d t z^{\nu+\mu+\lambda+\eta-1} \\
& \times(1-z)^{\frac{n-\nu-\mu-\lambda-\eta}{2}-1} y^{\mu-1}(1-y)^{\nu-1} t^{\nu+\mu+\lambda-1}(1-t)^{\eta-1} x^{\nu+\mu-1}(1-x)^{\lambda-1} \\
& \times\left(s_{1}-\frac{m_{1 Q}^{2}}{z x t(1-y)}-\frac{m_{2 Q}^{2}}{z x y t}-\frac{m_{3 Q}^{2}}{z t(1-x)}-\frac{m_{4 Q}^{2}}{z(1-t)}\right)^{\frac{n+\mu+\nu+\lambda+\eta}{2}+k-1} \\
& \times\left(\left(\frac{d}{d s_{1}}\right)^{k} \delta\left(s_{2}-s_{1}\right)\right) \theta\left(s_{1}-\frac{m_{1 Q}^{2}}{z x t(1-y)}-\frac{m_{2 Q}^{2}}{z x y t}-\frac{m_{3 Q}^{2}}{z t(1-x)}-\frac{m_{4 Q}^{2}}{z(1-t)} d 112\right)
\end{aligned}
$$

Using this spectral density, the continuum subtracted correlation function in the Borel scheme corresponding to the considered term can be written as

$$
\Pi_{4}^{s u b}\left(M_{1}^{2}, M_{2}^{2}\right)=\int_{s_{L}}^{s_{0}} d s_{1} \int_{s_{L}}^{s_{0}} d s_{2} \rho\left(s_{1}, s_{2}\right) e^{-s_{1} / M_{1}^{2}} e^{-s_{2} / M_{2}^{2}}
$$


where $s_{L}=\left(m_{1 Q}+m_{2 Q}+m_{3 Q}+m_{4 Q}\right)^{2}$. Defining new variables, $s_{1}=2 s v$ and $s_{2}=2 s(1-v)$, we get

$$
\Pi_{4}^{s u b}\left(M_{1}^{2}, M_{2}^{2}\right)=\int_{s_{L}}^{s_{0}} d s \int_{0}^{1} d v \rho\left(s_{1}, s_{2}\right)(4 s) e^{-2 s v / M_{1}^{2}} e^{-2 s(1-v) / M_{2}^{2}} .
$$

Using the expression for the spectral density, one can get

$$
\begin{aligned}
\Pi_{4}^{s u b}\left(M_{1}^{2}, M_{2}^{2}\right) & =\mathcal{C} \sum_{k} a_{k} \frac{(-1)^{k}}{\Gamma\left(\frac{n+\mu+\nu+\lambda+\eta}{2}+k\right)} \int_{s_{L}}^{s_{0}} d s \int_{0}^{1} d v \frac{1}{2^{k} s^{k}}\left(\left(\frac{d}{d v}\right)^{k} \delta(v-1 / 2)\right) \\
& \times \int_{0}^{4} d z \int_{0}^{1} d x \int_{0}^{1} d y \int_{0}^{1} d t z^{\nu+\mu+\lambda+\eta-1}(1-z)^{\frac{n-\nu-\mu-\lambda-\eta}{2}-1} \\
& \times y^{\mu-1}(1-y)^{\nu-1} t^{\nu+\mu+\lambda-1}(1-t)^{\eta-1} x^{\nu+\mu-1}(1-x)^{\lambda-1} \\
& \times\left(2 s v-\frac{m_{1 Q}^{2}}{z x t(1-y)}-\frac{m_{2 Q}^{2}}{z x y t}-\frac{m_{3 Q}^{2}}{z t(1-x)}-\frac{m_{4 Q}^{2}}{z(1-t)}\right)^{\frac{n+\mu+\nu+\lambda+\eta}{2}+k-1} \\
& \times \theta\left(2 s v-\frac{m_{1 Q}^{2}}{z x t(1-y)}-\frac{m_{2 Q}^{2}}{z x y t}-\frac{m_{3 Q}^{2}}{z t(1-x)}-\frac{m_{4 Q}^{2}}{z(1-t)}\right) e^{-2 s v / M_{1}^{2}} e^{-2 s(1-v) / M_{2}^{2}}
\end{aligned}
$$

Integrating over $v$, finally leads to

$$
\begin{aligned}
\Pi_{4}^{s u b}\left(M_{1}^{2}, M_{2}^{2}\right) & =(-i) \sum_{k} a_{k} \frac{2^{-n} \pi^{2}}{m_{1 Q}^{\nu} m_{2 Q}^{\mu} m_{3 Q}^{\lambda} m_{4 Q}^{\eta} \Gamma\left(\frac{n-\nu-\mu-\lambda-\eta}{2}\right) \Gamma\left(\frac{n+\mu+\nu+\lambda+\eta}{2}+k\right)} \int d s \frac{1}{2^{k} s^{k}} \\
& \times\left[\left(\frac{d}{d v}\right)^{k} \int_{0}^{4} d z \theta\left(2 s v-\frac{m_{1 Q}^{2}}{z x t(1-y)}-\frac{m_{2 Q}^{2}}{z x y t}-\frac{m_{3 Q}^{2}}{z t(1-x)}-\frac{m_{4 Q}^{2}}{z(1-t)}\right)\right. \\
& \times \int_{0}^{1} d x \int_{0}^{1} d y \int_{0}^{1} d t z^{\nu+\mu+\lambda+\eta-1}(1-z)^{\frac{n-\nu-\mu-\lambda-\eta}{2}-1} \\
& \times y^{\mu-1}(1-y)^{\nu-1} t^{\nu+\mu+\lambda-1}(1-t)^{\eta-1} x^{\nu+\mu-1}(1-x)^{\lambda-1} \\
& \times\left(2 s v-\frac{m_{1 Q}^{2}}{z x t(1-y)}-\frac{m_{2 Q}^{2}}{z x y t}-\frac{m_{3 Q}^{2}}{z t(1-x)}-\frac{m_{4 Q}^{2}}{z(1-t)}\right)^{\frac{n+\mu+\nu+\lambda+\eta}{2}+k-1} \\
& \left.\times e^{-2 s v / M_{1}^{2}} e^{-2 s(1-v) / M_{2}^{2}}\right]_{v=1 / 2}
\end{aligned}
$$

\subsection{Systems containing five heavy quarks}

The required function to be evaluated in this case is

$$
\begin{aligned}
T_{5} & =\int_{0}^{1} d u \int d^{4} x e^{i P . x} \frac{A(u)}{\left(\sqrt{-x^{2}}\right)^{n}} \\
& \times K_{\nu}\left(m_{1 Q} \sqrt{-x^{2}}\right) K_{\mu}\left(m_{2 Q} \sqrt{-x^{2}}\right) K_{\lambda}\left(m_{3 Q} \sqrt{-x^{2}}\right) K_{\eta}\left(m_{4 Q} \sqrt{-x^{2}}\right) K_{\xi}\left(m_{5 Q} \sqrt{-x^{2}}\right),
\end{aligned}
$$


where $m_{i Q}$, are the masses of heavy quarks. Using the integral representation of the modified Bessel function, we have

$$
\begin{aligned}
T_{5} & =\frac{\Gamma(\nu+1 / 2) \Gamma(\mu+1 / 2) \Gamma(\lambda+1 / 2) \Gamma(\eta+1 / 2) \Gamma(\xi+1 / 2) 2^{\mu+\nu+\lambda+\eta+\xi}}{(\pi)^{5 / 2} m_{1 Q}^{\nu} m_{2 Q}^{\mu} m_{3 Q}^{\lambda} m_{4 Q}^{\eta} m_{5 Q}^{\xi}} \\
& \times \int_{0}^{\infty} d t_{5} \int_{0}^{\infty} d t_{4} \int_{0}^{\infty} d t_{3} \int_{0}^{\infty} d t_{2} \int_{0}^{\infty} d t_{1} \int_{0}^{1} d u \int d^{4} x e^{i P \cdot x} A(u) \\
& \times \frac{\cos \left(m_{1 Q} t_{1}\right) \cos \left(m_{2 Q} t_{2}\right) \cos \left(m_{3 Q} t_{3}\right) \cos \left(m_{4 Q} t_{4}\right) \cos \left(m_{5 Q} t_{5}\right)}{\left(t_{1}^{2}-x^{2}\right)^{\nu+1 / 2}\left(t_{2}^{2}-x^{2}\right)^{\mu+1 / 2}\left(t_{3}^{2}-x^{2}\right)^{\lambda+1 / 2}\left(t_{4}^{2}-x^{2}\right)^{\eta+1 / 2}\left(t_{5}^{2}-x^{2}\right)^{\xi+1 / 2}\left(-x^{2}\right)^{\frac{n-\mu-\nu-\lambda-\eta-\xi}{2}}} .
\end{aligned}
$$

In this step, for further calculations, we again perform a Wick rotation to go the Euclidean space and use the Schwinger representation. We get

$$
\begin{aligned}
T_{5} & =\frac{(-i) 2^{\mu+\nu+\lambda+\eta+\xi}}{(\pi)^{5 / 2} m_{1 Q}^{\nu} m_{2 Q}^{\mu} m_{3 Q}^{\lambda} m_{4 Q}^{\eta} m_{5 Q}^{\xi} \Gamma\left(\frac{n-\mu-\nu-\lambda-\eta-\xi}{2}\right)} \int_{0}^{1} d u \int d^{4} x e^{-i P \cdot x} A(u) \int_{0}^{\infty} d t_{5} \int_{0}^{\infty} d t_{4} \int_{0}^{\infty} d t_{3} \\
& \times \int_{0}^{\infty} d t_{2} \int_{0}^{\infty} d t_{1} \int_{0}^{\infty} d y_{5} \int_{0}^{\infty} d y_{4} \int_{0}^{\infty} d y_{3} \int_{0}^{\infty} d y_{2} \int_{0}^{\infty} d y_{1} \int_{0}^{\infty} d y_{0} y_{0}^{\frac{n-\mu-\nu-\lambda-\eta-\xi}{2}}-1 e^{-y_{0} x^{2}} \\
& \times y_{1}^{\nu-\frac{1}{2}} e^{-y_{1}\left(x^{2}+t_{1}^{2}\right)} y_{2}^{\mu-\frac{1}{2}} e^{-y_{2}\left(x^{2}+t_{2}^{2}\right)} y_{3}^{\lambda-\frac{1}{2}} e^{-y_{3}\left(x^{2}+t_{3}^{2}\right)} y_{4}^{\eta-\frac{1}{2}} e^{-y_{4}\left(x^{2}+t_{4}^{2}\right)} y_{5}^{\xi-\frac{1}{2}} e^{-y_{5}\left(x^{2}+t_{5}^{2}\right)} \\
& \times \cos \left(m_{1 Q} t_{1}\right) \cos \left(m_{2 Q} t_{2}\right) \cos \left(m_{3 Q} t_{3}\right) \cos \left(m_{4 Q} t_{4}\right) \cos \left(m_{5 Q} t_{5}\right) .
\end{aligned}
$$

The next step is to perform the Gaussian integral over $x$ and integrate over $t_{i}$ 's. This leads to

$$
\begin{aligned}
T_{5} & =(-i) \frac{2^{\mu+\nu+\lambda+\eta+\xi-5} \pi^{2}}{m_{1 Q}^{\nu} m_{2 Q}^{\mu} m_{3 Q}^{\lambda} m_{4 Q}^{\eta} m_{5 Q}^{\xi} \Gamma\left(\frac{n-\mu-\nu-\lambda-\eta-\xi}{2}\right)} \int_{0}^{1} d u A(u) \int_{0}^{\infty} d y_{5} \int_{0}^{\infty} d y_{4} \int_{0}^{\infty} d y_{3} \\
& \times \int_{0}^{\infty} d y_{2} \int_{0}^{\infty} d y_{1} \int_{0}^{\infty} d y_{0} e^{\frac{-P^{2}}{4\left(y_{0}+y_{1}+y_{2}+y_{3}+y_{4}+y_{5}\right)}} y_{0}^{\frac{n-\mu-\nu-\lambda-\eta-\xi}{2}}-1 \\
& \times \frac{y_{1}^{\nu-1} e^{-\frac{m_{1 Q}^{2}}{4 y_{1}}} y_{2}^{\mu-1} e^{-\frac{m_{2 Q}^{2}}{4 y_{2}}} y_{3}^{\lambda-1} e^{-\frac{m_{3 Q}^{2}}{4 y_{3}}} y_{4}^{\eta-1} e^{-\frac{m_{4 Q}^{2}}{4 y_{4}}} y_{5}^{\xi-1} e^{-\frac{m_{5 Q}^{2}}{4 y_{5}}}}{\left(y_{0}+y_{1}+y_{2}+y_{3}+y_{4}+y_{5}\right)^{2}} .
\end{aligned}
$$

We introduce the variables $\rho, v, l, r, h$ and $w$, defined by

$$
\begin{aligned}
\rho & =y_{1}+y_{2}+y_{3}+y_{4}+y_{5}, & v & =\frac{y_{1}}{y_{1}+y_{2}+y_{3}+y_{4}+y_{5}}, \\
r & =\frac{y_{2}}{y_{1}+y_{2}+y_{3}+y_{4}}, & w & =\frac{y_{3}}{y_{1}+y_{2}+y_{3}+y_{4}+y_{5}}, \\
l & =\frac{y_{5}}{y_{1}+y_{2}+y_{3}+y_{4}+y_{5}}, & h & =\frac{y_{5}}{y_{1}+y_{2}+y_{3}+y_{4}+y_{5}},
\end{aligned}
$$

to write the function $T_{5}$ as

$$
\begin{aligned}
T_{5} & =(-i) \frac{2^{\mu+\nu+\lambda+\eta+\xi-5} \pi^{2}}{m_{1 Q}^{\nu} m_{2 Q}^{\mu} m_{3 Q}^{\lambda} m_{4 Q}^{\eta} m_{5 Q}^{\xi} \Gamma\left(\frac{n-\mu-\nu-\lambda-\eta-\xi}{2}\right)} \int_{0}^{1} d u A(u) \int_{0}^{1} d w \int_{0}^{1} d v \int_{0}^{1} d r \\
& \times \int_{0}^{1} d l \int_{0}^{1} d h \int_{0}^{\infty} \rho^{3} d \rho e^{\frac{-P^{2}}{4 \rho}}(\rho(1-v-r-l-w-h))^{\frac{n-\mu-\nu-\lambda-\eta-\xi}{2}}-1 \\
& \times(\rho v)^{\nu-1} e^{-\frac{m_{1 Q}^{2}}{4 \rho v}}(\rho r)^{\mu-1} e^{-\frac{m_{2 Q}^{2}}{4 \rho r}}(\rho w)^{\lambda-1} e^{-\frac{m_{3 Q}^{2}}{4 \rho w}}(\rho l)^{\eta-1} e^{-\frac{m_{4 Q}^{2}}{4 \rho l}}(\rho h)^{\xi-1} e^{-\frac{m_{5 Q}^{2}}{4 \rho h}}
\end{aligned}
$$


Now we apply the double Borel transformation and perform the integrals over $u$ and $\rho$,

$$
\begin{aligned}
\mathcal{B}_{M_{1}} \mathcal{B}_{M_{2}} T_{5} & =(-i) \frac{2^{-1-n} \pi^{2}}{m_{1 Q}^{\nu} m_{2 Q}^{\mu} m_{3 Q}^{\lambda} m_{4 Q}^{\eta} m_{5 Q}^{\xi} \Gamma\left(\frac{n-\mu-\nu-\lambda-\eta-\xi}{2}\right)} A\left(u_{0}\right)\left(M^{2}\right)^{\frac{n+\mu+\nu+\lambda+\eta+\xi}{2}} \\
& \times \int_{0}^{1} d w \int_{0}^{1} d v \int_{0}^{1} d r \int_{0}^{1} d l \int_{0}^{1} d h(1-v-r-l-w-h)^{\frac{n-\mu-\nu-\lambda-\eta-\xi}{2}}-1 \\
& \times v^{\nu-1} e^{-\frac{m_{1 Q}^{2}}{M^{2} v}} r^{\mu-1} e^{-\frac{m_{2 Q}^{2}}{M^{2} r}} w^{\lambda-1} e^{-\frac{m_{3 Q}^{2}}{M^{2} w}} l^{\eta-1} e^{-\frac{m_{4 Q}^{2}}{M^{2} l}} h^{\xi-1} e^{-\frac{m_{5 Q}^{2}}{M^{2} h}} e^{\frac{q^{2}}{M_{1}^{2}+M_{2}^{2}}} .
\end{aligned}
$$

The following new set of variables make the function $\mathcal{B}_{M_{1}} \mathcal{B}_{M_{2}} T$ easy to process:

$$
v=z x t j(1-y), \quad r=z x y t j, \quad w=z t j(1-x), \quad l=z j(1-t), \quad h=z(1-j),
$$

so,we get

$$
\begin{aligned}
\mathcal{B}_{M_{1}} \mathcal{B}_{M_{2}} T_{5} & =(-i) \frac{2^{-1-n} \pi^{2}}{m_{1 Q}^{\nu} m_{2 Q}^{\mu} m_{3 Q}^{\lambda} m_{4 Q}^{\eta} m_{5 Q}^{\xi} \Gamma\left(\frac{n-\mu-\nu-\lambda-\eta-\xi}{2}\right)} A\left(u_{0}\right)\left(M^{2}\right)^{\frac{n+\mu+\nu+\lambda+\eta+\xi}{2}} \\
& \times \int_{0}^{5} d z \int_{0}^{1} d x \int_{0}^{1} d y \int_{0}^{1} d t \int_{0}^{1} d l \int_{0}^{1} d j z^{\nu+\mu+\lambda+\eta+\xi-1}(1-z)^{\frac{n-\nu-\mu-\lambda-\eta-\xi}{2}-1} \\
& \times j^{\nu+\mu+\lambda+\eta-1}(1-j)^{\xi-1} t^{\nu+\mu+\lambda-1}(1-t)^{\eta-1} x^{\nu+\mu-1}(1-x)^{\lambda-1} y^{\mu-1}(1-y)^{\nu-1} \\
& \times e^{-\frac{m_{1 Q}^{2}}{M^{2} z x t j(1-y)}} e^{-\frac{m_{2 Q}^{2}}{M^{2} z x y t j}} e^{-\frac{m_{3 Q}^{2}}{M^{2} z t j(1-x)}} e^{-\frac{m_{4 Q}^{2}}{M^{2} z j(1-t)}} e^{-\frac{m_{5 Q}^{2}}{M^{2} z(1-j)}}
\end{aligned}
$$

\section{Spectral representation and continuum subtraction for systems containing five heavy quarks}

We take the general term

$$
\begin{aligned}
\Pi_{5} & =\mathcal{C}\left(M^{2}\right)^{\frac{n+\mu+\nu+\lambda+\eta+\xi}{2}} A\left(u_{0}\right) \int_{0}^{5} d z \int_{0}^{1} d x \int_{0}^{1} d y \int_{0}^{1} d t \\
& \times \int_{0}^{1} d j z^{\nu+\mu+\lambda+\eta+\xi-1}(1-z)^{\frac{n-\nu-\mu-\lambda-\eta-\xi}{2}-1} \\
& \times j^{\nu+\mu+\lambda+\eta-1}(1-j)^{\xi-1} t^{\nu+\mu+\lambda-1}(1-t)^{\eta-1} x^{\nu+\mu-1}(1-x)^{\lambda-1} y^{\mu-1}(1-y)^{\nu-1} \\
& \times e^{-\frac{m_{1 Q}^{2}}{M^{2} z x t j(1-y)}} e^{-\frac{m_{2 Q}^{2}}{M^{2} z x y t j}} e^{-\frac{m_{3 Q}^{2}}{M^{2} z t j(1-x)}} e^{-\frac{m_{4 Q}^{2}}{M^{2} z j(1-t)}} e^{-\frac{m_{5 Q}^{2}}{M^{2} z(1-j)}},
\end{aligned}
$$

where

$$
\mathcal{C}=(-i) \frac{2^{-1-n} \pi^{2}}{m_{1 Q}^{\nu} m_{2 Q}^{\mu} m_{3 Q}^{\lambda} m_{4 Q}^{\eta} m_{5 Q}^{\xi} \Gamma\left(\frac{n-\mu-\nu-\lambda-\eta-\xi}{2}\right)} .
$$

With expanding $A\left(u_{0}\right)$, this leads to 


$$
\begin{aligned}
\Pi_{5} & =\mathcal{C} \sum_{k} a_{k} \frac{(-1)^{k}}{\Gamma\left(\frac{n+\mu+\nu+\lambda+\eta+\xi}{2}+k\right)} \int_{0}^{5} d z \int_{0}^{1} d x \int_{0}^{1} d y \int_{0}^{1} d t \\
& \times \int_{0}^{1} d j z^{\nu+\mu+\lambda+\eta+\xi-1}(1-z)^{\frac{n-\nu-\mu-\lambda-\eta-\xi}{2}}-1 j^{\nu+\mu+\lambda+\eta-1}(1-j)^{\xi-1} \\
& \times t^{\nu+\mu+\lambda-1}(1-t)^{\eta-1} x^{\nu+\mu-1}(1-x)^{\lambda-1} y^{\mu-1}(1-y)^{\nu-1} \\
& \times \int_{0}^{\infty} d l l^{\frac{n+\mu+\nu+\lambda+\eta+\xi}{2}+k-1} e^{-\left(l+\frac{m_{1 Q}^{2}}{z x t j(1-y)}+\frac{m_{2 Q}^{2}}{z x y t j}+\frac{m_{3 Q}^{2}}{z t j(1-x)}+\frac{m_{4 Q}^{2}}{z j(1-t)}+\frac{m_{5 Q}^{2}}{z(1-j)}\right) \sigma_{2}} \\
& \times\left(\left(\frac{d}{d l}\right)^{k} e^{-\left(l+\frac{m_{1 Q}^{2}}{z x t j(1-y)}+\frac{m_{2 Q}^{2}}{z x y t j}+\frac{m_{3 Q}^{2}}{z t j(1-x)}+\frac{m_{4 Q}^{2}}{z j(1-t)}+\frac{m_{5 Q}^{2}}{z(1-j)}\right) \sigma_{1}}\right) .
\end{aligned}
$$

By applying double Borel transformation with respect to $\sigma_{1} \rightarrow \frac{1}{\tau_{1}}$ and $\sigma_{2} \rightarrow \frac{1}{\tau_{2}}$, we obtain

$$
\begin{aligned}
\mathcal{B}_{1 / \tau_{1}} \mathcal{B}_{1 / \tau_{2}} \Pi_{5} & =\mathcal{C} \sum_{k} a_{k} \frac{(-1)^{k}}{\Gamma\left(\frac{n+\mu+\nu+\lambda+\eta+\xi}{2}+k\right)} \int_{0}^{\infty} d l l^{\frac{n+\mu+\nu+\lambda+\eta+\xi}{2}+k-1} \\
& \times \int_{0}^{5} d z \int_{0}^{1} d x \int_{0}^{1} d y \int_{0}^{1} d t \int_{0}^{1} d j z^{\nu+\mu+\lambda+\eta+\xi-1}(1-z)^{\frac{n-\nu-\mu-\lambda-\eta-\xi}{2}}-1 \\
& \times j^{\nu+\mu+\lambda+\eta-1}(1-j)^{\xi-1} t^{\nu+\mu+\lambda-1}(1-t)^{\eta-1} x^{\nu+\mu-1}(1-x)^{\lambda-1} y^{\mu-1}(1-y)^{\nu-1} \\
& \times\left(\left(\frac{d}{d l}\right)^{k} \delta\left(\tau_{1}-\left(l+\frac{m_{1 Q}^{2}}{z x t j(1-y)}+\frac{m_{2 Q}^{2}}{z x y t j}+\frac{m_{3 Q}^{2}}{z t j(1-x)}+\frac{m_{4 Q}^{2}}{z j(1-t)}+\frac{m_{5 Q}^{2}}{z(1-j)}\right)\right)\right. \\
& \times \delta\left(\tau_{2}-\left(l+\frac{m_{1 Q}^{2}}{z x t j(1-y)}+\frac{m_{2 Q}^{2}}{z x y t j}+\frac{m_{3 Q}^{2}}{z t j(1-x)}+\frac{m_{4 Q}^{2}}{z j(1-t)}+\frac{m_{5 Q}^{2}}{z(1-j)}\right)\right),
\end{aligned}
$$

and this leads to the spectral density

$$
\begin{aligned}
\rho_{5}\left(s_{1}, s_{2}\right) & =\mathcal{C} \sum_{k} a_{k} \frac{(-1)^{k}}{\Gamma\left(\frac{n+\mu+\nu+\lambda+\eta+\xi}{2}+k\right)} \int_{0}^{\infty} d l l^{\frac{n+\mu+\nu+\lambda+\eta+\xi}{2}+k-1} \\
& \times \int_{0}^{5} d z \int_{0}^{1} d x \int_{0}^{1} d y \int_{0}^{1} d t \int_{0}^{1} d j z^{\nu+\mu+\lambda+\eta+\xi-1}(1-z)^{\frac{n-\nu-\mu-\lambda-\eta-\xi}{2}}-1 \\
& \times j^{\nu+\mu+\lambda+\eta-1}(1-j)^{\xi-1} t^{\nu+\mu+\lambda-1}(1-t)^{\eta-1} x^{\nu+\mu-1}(1-x)^{\lambda-1} y^{\mu-1}(1-y)^{\nu-1} \\
& \times\left(\left(\frac{d}{d l}\right)^{k} \delta\left(s_{1}-\left(l+\frac{m_{1 Q}^{2}}{z x t j(1-y)}+\frac{m_{2 Q}^{2}}{z x y t j}+\frac{m_{3 Q}^{2}}{z t j(1-x)}+\frac{m_{4 Q}^{2}}{z j(1-t)}+\frac{m_{5 Q}^{2}}{z(1-j)}\right)\right)\right. \\
& \times \delta\left(s_{2}-\left(l+\frac{m_{1 Q}^{2}}{z x t j(1-y)}+\frac{m_{2 Q}^{2}}{z x y t j}+\frac{m_{3 Q}^{2}}{z t j(1-x)}+\frac{m_{4 Q}^{2}}{z j(1-t)}+\frac{m_{5 Q}^{2}}{z(1-j)}\right)\right) .
\end{aligned}
$$

Performing the integration over $l$, finally we obtain the following expression for the double 
spectral density:

$$
\begin{aligned}
\rho_{5}\left(s_{1}, s_{2}\right) & =\mathcal{C} \sum_{k} a_{k} \frac{(-1)^{k}}{\Gamma\left(\frac{n+\mu+\nu+\lambda+\eta+\xi}{2}+k\right)} \int_{0}^{5} d z \int_{0}^{1} d x \int_{0}^{1} d y \int_{0}^{1} d t \\
& \times \int_{0}^{1} d j z^{\nu+\mu+\lambda+\eta+\xi-1}(1-z)^{\frac{n-\nu-\mu-\lambda-\eta-\xi}{2}-1} \\
& \times j^{\nu+\mu+\lambda+\eta-1}(1-j)^{\xi-1} t^{\nu+\mu+\lambda-1}(1-t)^{\eta-1} x^{\nu+\mu-1}(1-x)^{\lambda-1} y^{\mu-1}(1-y)^{\nu-1} \\
& \times\left(s_{1}-\left(\frac{m_{1 Q}^{2}}{z x t j(1-y)}+\frac{m_{2 Q}^{2}}{z x y t j}+\frac{m_{3 Q}^{2}}{z t j(1-x)}+\frac{m_{4 Q}^{2}}{z j(1-t)}+\frac{m_{5 Q}^{2}}{z(1-j)}\right)\right) \\
& \times \theta\left(s_{1}-\left(\frac{m_{1 Q}^{2}}{z x t j(1-y)}+\frac{m_{2 Q}^{2}}{z x y t j}+\frac{m_{3 Q}^{2}}{z t j(1-x)}+\frac{m_{4 Q}^{2}}{z j(1-t)}+\frac{m_{5 Q}^{2}}{z(1-j)}\right)\right) \\
& \times\left(\left(\frac{d}{d s_{1}}\right)^{k} \delta\left(s_{2}-s_{1}\right)\right)
\end{aligned}
$$

Using this spectral density, the continuum subtracted correlation function in the Borel scheme corresponding to the considered term can be written as:

$$
\Pi_{5}^{s u b}\left(M_{1}^{2}, M_{2}^{2}\right)=\int_{s_{L}}^{s_{0}} d s_{1} \int_{s_{L}}^{s_{0}} d s_{2} \rho\left(s_{1}, s_{2}\right) e^{-s_{1} / M_{1}^{2}} e^{-s_{2} / M_{2}^{2}}
$$

where $s_{L}=\left(m_{1 Q}+m_{2 Q}+m_{3 Q}+m_{4 Q}+m_{5 Q}\right)^{2}$. Defining new variables, $s_{1}=2 s v$ and $s_{2}=2 s(1-v)$, we get

$$
\Pi_{5}^{s u b}\left(M_{1}^{2}, M_{2}^{2}\right)=\int_{s_{L}}^{s_{0}} d s \int_{0}^{1} d v \rho\left(s_{1}, s_{2}\right)(4 s) e^{-2 s v / M_{1}^{2}} e^{-2 s(1-v) / M_{2}^{2}} .
$$

Using the expression for the spectral density, one can get

$$
\begin{aligned}
\Pi_{5}^{s u b}\left(M_{1}^{2}, M_{2}^{2}\right) & =\mathcal{C} \sum_{k} a_{k} \frac{(-1)^{k}}{\Gamma\left(\frac{n+\mu+\nu+\lambda+\eta+\xi}{2}+k\right)} \int_{s_{L}}^{s_{0}} d s \\
& \times \int_{0}^{1} d v \frac{1}{2^{k} s^{k}}\left(\left(\frac{d}{d v}\right)^{k} \delta(v-1 / 2)\right) e^{-2 s v / M_{1}^{2}} e^{-2 s(1-v) / M_{2}^{2}} \\
& \times \int_{0}^{5} d z \int_{0}^{1} d x \int_{0}^{1} d y \int_{0}^{1} d t \int_{0}^{1} d j z^{\nu+\mu+\lambda+\eta+\xi-1}(1-z)^{\frac{n-\nu-\mu-\lambda-\eta-\xi}{2}-1} \\
& \times j^{\nu+\mu+\lambda+\eta-1}(1-j)^{\xi-1} t^{\nu+\mu+\lambda-1}(1-t)^{\eta-1} x^{\nu+\mu-1}(1-x)^{\lambda-1} y^{\mu-1}(1-y)^{\nu-1} \\
& \times\left(2 s v-\left(\frac{m_{1 Q}^{2}}{z x t j(1-y)}+\frac{m_{2 Q}^{2}}{z x y t j}+\frac{m_{3 Q}^{2}}{z t j(1-x)}+\frac{m_{4 Q}^{2}}{z j(1-t)}+\frac{m_{5 Q}^{2}}{z(1-j)}\right)\right) \\
& \times \theta\left(2 s v-\left(\frac{m_{1 Q}^{2}}{z x t j(1-y)}+\frac{m_{2 Q}^{2}}{z x y t j}+\frac{m_{3 Q}^{2}}{z t j(1-x)}+\frac{m_{4 Q}^{2}}{z j(1-t)}+\frac{m_{5 Q}^{2}}{z(1-j)}\right)\right) .
\end{aligned}
$$


By integrating over $v$, finally we obtain

$$
\begin{aligned}
\Pi_{5}^{s u b}\left(M_{1}^{2}, M_{2}^{2}\right) & =\sum_{k} a_{k} \frac{(-i) 2^{-1-n} \pi^{2}}{m_{1 Q}^{\nu} m_{2 Q}^{\mu} m_{3 Q}^{\lambda} m_{4 Q}^{\eta} m_{5 Q}^{\xi} \Gamma\left(\frac{n-\mu-\nu-\lambda-\eta-\xi}{2}\right) \Gamma\left(\frac{n+\mu+\nu+\lambda+\eta+\xi}{2}+k\right)} \int_{s_{L}}^{s_{0}} d s \frac{1}{2^{k} s^{k}} \\
& \times\left[\left(\frac{d}{d v}\right)^{k} \int_{0}^{5} d z \theta\left(2 s v-\left(\frac{m_{1 Q}^{2}}{z x t j(1-y)}+\frac{m_{2 Q}^{2}}{z x y t j}+\frac{m_{3 Q}^{2}}{z t j(1-x)}+\frac{m_{4 Q}^{2}}{z j(1-t)}+\frac{m_{5 Q}^{2}}{z(1-j)}\right)\right)\right. \\
& \times \int_{0}^{1} d x \int_{0}^{1} d y \int_{0}^{1} d t \int_{0}^{1} d j z^{\nu+\mu+\lambda+\eta+\xi-1}(1-z)^{\frac{n-\nu-\mu-\lambda-\eta-\xi}{2}-1} \\
& \times j^{\nu+\mu+\lambda+\eta-1}(1-j)^{\xi-1} t^{\nu+\mu+\lambda-1}(1-t)^{\eta-1} x^{\nu+\mu-1}(1-x)^{\lambda-1} y^{\mu-1}(1-y)^{\nu-1} \\
& \times\left(2 s v-\left(\frac{m_{1 Q}^{2}}{z x t j(1-y)}+\frac{m_{2 Q}^{2}}{z x y t j}+\frac{m_{3 Q}^{2}}{z t j(1-x)}+\frac{m_{4 Q}^{2}}{z j(1-t)}+\frac{m_{5 Q}^{2}}{z(1-j)}\right)\right)^{\frac{n+\mu+\nu+\lambda+\eta+\xi}{2}+k-1} \\
& \times e^{\left.-2 s v / M_{1}^{2} e^{-2 s(1-v) / M_{2}^{2}}\right]_{v=1 / 2}}
\end{aligned}
$$

\section{Conclusion}

We worked out the mathematics required for the calculations of the parameters related to the spectroscopy as well as the electromagnetic, weak and strong decays of the light and heavy systems with two-five heavy $b$ or $c$ quarks. In particular, we presented the calculations required in the Fourier and Borel transformations as well as continuum subtraction of the considered systems. We showed that by choosing an appropriate representation of the modified Bessel functions of the second kind and applying successive Borel transformations with the aim of more suppression of the unwanted contributions, how we can get finite results without any divergence. Such divergences are appeared for systems of two heavy quarks by choosing a special integral form of the modified Bessel functions and performing the regular calculations according to the standard prescriptions of the QCD sum rule approach as done in the Appendix $\mathrm{C}$ of the Ref. [48.

The method presented here greatly simplifies the calculations of the correlation functions for systems containing more than two heavy quarks. Our results can be used in calculations of many parameters of the conventional and non-conventional heavy hadrons and their interactions with other particles using the non-perturbative approaches like QCD sum rules.

\section{Acknowledgments}

S. R. and A. R. O. would like to thank School of Physics, Institute for Research in Fundamental Sciences (IPM) for their hospitality where a part of this work was performed. K. A. thanks Doğuş University for the partial financial support through the grant BAP 2015-16-D1-B04.

\section{Appendix: DAs of pseudoscalar mesons}

In this Appendix, we present the matrix elements $\langle\mathcal{P}(q)|\bar{q}(x) \Gamma q(0)| 0\rangle$ and $\left\langle\mathcal{P}(q)\left|\bar{q}(x) \Gamma G_{\mu \nu} q(0)\right| 0\right\rangle$ representing the interactions of the particles under consideration in terms of the wave functions 
of pseudoscalar mesons [49 51]:

$$
\begin{aligned}
& \left\langle\mathcal{P}(q)\left|\bar{q}(x) \gamma_{\mu} \gamma_{5} q(0)\right| 0\right\rangle=-i f_{\mathcal{P}} q_{\mu} \int_{0}^{1} d u e^{i \bar{u} q x}\left(\varphi_{\mathcal{P}}(u)+\frac{1}{16} m_{\mathcal{P}}^{2} x^{2} \mathbb{A}(u)\right) \\
& -\frac{i}{2} f_{\mathcal{P}} m_{\mathcal{P}}^{2} \frac{x_{\mu}}{q x} \int_{0}^{1} d u e^{i \bar{u} q x} \mathbb{B}(u), \\
& \left\langle\mathcal{P}(q)\left|\bar{q}(x) i \gamma_{5} q(0)\right| 0\right\rangle=\mu_{\mathcal{P}} \int_{0}^{1} d u e^{i \bar{u} q x} \varphi_{P}(u) \\
& \left\langle\mathcal{P}(q)\left|\bar{q}(x) \sigma_{\alpha \beta} \gamma_{5} q(0)\right| 0\right\rangle=\frac{i}{6} \mu_{\mathcal{P}}\left(1-\widetilde{\mu}_{\mathcal{P}}^{2}\right)\left(q_{\alpha} x_{\beta}-q_{\beta} x_{\alpha}\right) \int_{0}^{1} d u e^{i \bar{u} q x} \varphi_{\sigma}(u), \\
& \left\langle\mathcal{P}(q)\left|\bar{q}(x) \sigma_{\mu \nu} \gamma_{5} g_{s} G_{\alpha \beta}(v x) q(0)\right| 0\right\rangle=i \mu_{\mathcal{P}}\left[q_{\alpha} q_{\mu}\left(g_{\nu \beta}-\frac{1}{q x}\left(q_{\nu} x_{\beta}+q_{\beta} x_{\nu}\right)\right)\right. \\
& -q_{\alpha} q_{\nu}\left(g_{\mu \beta}-\frac{1}{q x}\left(q_{\mu} x_{\beta}+q_{\beta} x_{\mu}\right)\right) \\
& -q_{\beta} q_{\mu}\left(g_{\nu \alpha}-\frac{1}{q x}\left(q_{\nu} x_{\alpha}+q_{\alpha} x_{\nu}\right)\right) \\
& \left.+q_{\beta} q_{\nu}\left(g_{\mu \alpha}-\frac{1}{q x}\left(q_{\mu} x_{\alpha}+q_{\alpha} x_{\mu}\right)\right)\right] \\
& \times \int D \alpha e^{i\left(\alpha_{\bar{q}}+v \alpha_{g}\right) q x} \mathcal{T}\left(\alpha_{i}\right), \\
& \left\langle\mathcal{P}(q)\left|\bar{q}(x) \gamma_{\mu} \gamma_{5} g_{s} G_{\alpha \beta}(v x) q(0)\right| 0\right\rangle=q_{\mu}\left(q_{\alpha} x_{\beta}-q_{\beta} x_{\alpha}\right) \frac{1}{q x} f_{\mathcal{P}} m_{\mathcal{P}}^{2} \int D \alpha e^{i\left(\alpha_{\bar{q}}+v \alpha_{g}\right) q x} \mathcal{A}_{\|}\left(\alpha_{i}\right) \\
& +\left[q_{\beta}\left(g_{\mu \alpha}-\frac{1}{q x}\left(q_{\mu} x_{\alpha}+q_{\alpha} x_{\mu}\right)\right)\right. \\
& \left.-q_{\alpha}\left(g_{\mu \beta}-\frac{1}{q x}\left(q_{\mu} x_{\beta}+q_{\beta} x_{\mu}\right)\right)\right] f_{\mathcal{P}} m_{\mathcal{P}}^{2} \\
& \times \int D \alpha e^{i\left(\alpha_{\bar{q}}+v \alpha_{g}\right) q x} \mathcal{A}_{\perp}\left(\alpha_{i}\right), \\
& \left\langle\mathcal{P}(q)\left|\bar{q}(x) \gamma_{\mu} i g_{s} G_{\alpha \beta}(v x) q(0)\right| 0\right\rangle=q_{\mu}\left(q_{\alpha} x_{\beta}-q_{\beta} x_{\alpha}\right) \frac{1}{q x} f_{\mathcal{P}} m_{\mathcal{P}}^{2} \int D \alpha e^{i\left(\alpha_{\bar{q}}+v \alpha_{g}\right) q x} \mathcal{V}_{\|}\left(\alpha_{i}\right) \\
& +\left[q_{\beta}\left(g_{\mu \alpha}-\frac{1}{q x}\left(q_{\mu} x_{\alpha}+q_{\alpha} x_{\mu}\right)\right)\right. \\
& \left.-q_{\alpha}\left(g_{\mu \beta}-\frac{1}{q x}\left(q_{\mu} x_{\beta}+q_{\beta} x_{\mu}\right)\right)\right] f_{\mathcal{P}} m_{\mathcal{P}}^{2} \\
& \times \int D \alpha e^{i\left(\alpha_{\bar{q}}+v \alpha_{g}\right) q x} \mathcal{V}_{\perp}\left(\alpha_{i}\right) \text {. }
\end{aligned}
$$

In Eq. (136) we have,

$$
\mu_{\mathcal{P}}=f_{\mathcal{P}} \frac{m_{\mathcal{P}}^{2}}{m_{q_{1}}+m_{q_{2}}}, \quad \tilde{\mu}_{\mathcal{P}}=\frac{m_{q_{1}}+m_{q_{2}}}{m_{\mathcal{P}}},
$$

and $D \alpha=d \alpha_{\bar{q}} d \alpha_{q} d \alpha_{g} \delta\left(1-\alpha_{\bar{q}}-\alpha_{q}-\alpha_{g}\right)$, and and the DA's $\varphi_{\mathcal{P}}(u), \mathbb{A}(u), \mathbb{B}(u), \varphi_{P}(u)$, $\varphi_{\sigma}(u), \mathcal{T}\left(\alpha_{i}\right), \mathcal{A}_{\perp}\left(\alpha_{i}\right), \mathcal{A}_{\|}\left(\alpha_{i}\right), \mathcal{V}_{\perp}\left(\alpha_{i}\right)$ and $\mathcal{V}_{\|}\left(\alpha_{i}\right)$ are functions of definite twist whose explicit expressions can be found in [49 51 ].

\section{References}

[1] T. M. Aliev, K. Azizi and H. Sundu, "Analysis of the structure of $\Xi(1690)$ through its decays," Eur. Phys. J. C 78, no. 5, 396 (2018) [arXiv:1804.02656 [hep-ph]]. 
[2] T. M. Aliev, K. Azizi and H. Sundu, "On the nature of $\Xi_{c}(2930)$," arXiv:1803.04002 [hep-ph].

[3] K. Azizi and N. Er, "Properties of $\Sigma_{Q}^{*}, \Xi_{Q}^{*}$ and $\Omega_{Q}^{*}$ heavy baryons in cold nuclear matter," Nucl. Phys. A 970, 422 (2018) [arXiv:1801.02168 [hep-ph]].

[4] S. S. Agaev, K. Azizi and H. Sundu, "Interpretation of the new $\Omega_{c}^{0}$ states via their mass and width," Eur. Phys. J. C 77, no. 6, 395 (2017) [arXiv:1704.04928 [hep-ph]].

[5] M. Mattson et al. [SELEX Collaboration], "First observation of the doubly charmed baryon Xi+(cc)," Phys. Rev. Lett. 89, 112001 (2002) [hep-ex/0208014].

[6] A. Ocherashvili et al. [SELEX Collaboration], "Confirmation of the double charm baryon $\mathrm{Xi}+(\mathrm{cc})(3520)$ via its decay to p D+ K-," Phys. Lett. B 628, 18 (2005) [hep-ex/0406033].

[7] R. Aaij et al. [LHCb Collaboration], "Observation of the doubly charmed baryon $\Xi_{c c}^{++}, "$ Phys. Rev. Lett. 119, no. 11, 112001 (2017) [arXiv:1707.01621 [hep-ex]].

[8] R. L. Jaffe, "Multi-Quark Hadrons. 1. The Phenomenology of (2 Quark 2 anti-Quark) Mesons," Phys. Rev. D 15, 267 (1977).

[9] R. L. Jaffe, "Multi-Quark Hadrons. 2. Methods," Phys. Rev. D 15, 281 (1977).

[10] N. Isgur and J. E. Paton, "A Flux Tube Model for Hadrons in QCD," Phys. Rev. D 31, $2910(1985)$.

[11] A. De Rujula, H. Georgi and S. L. Glashow, "Molecular Charmonium: A New Spectroscopy?," Phys. Rev. Lett. 38, 317 (1977).

[12] R. L. Jaffe, "Exotica," Phys. Rept. 409, 1 (2005) [hep-ph/0409065].

[13] R. L. Jaffe and K. Johnson, "Unconventional States of Confined Quarks and Gluons," Phys. Lett. 60B, 201 (1976).

[14] S. K. Choi et al. [Belle Collaboration], "Observation of a narrow charmonium - like state in exclusive $B^{ \pm} \rightarrow K^{ \pm} \pi^{+} \pi^{-} J / \psi$ decays," Phys. Rev. Lett. 91, 262001 (2003) [hepex/0309032].

[15] R. Aaij et al. [LHCb Collaboration], "Observation of $J / \psi p$ Resonances Consistent with Pentaquark States in $\Lambda_{b}^{0} \rightarrow J / \psi K^{-} p$ Decays," Phys. Rev. Lett. 115, 072001 (2015) [arXiv:1507.03414 [hep-ex]].

[16] K. Azizi, Y. Sarac and H. Sundu, "Strong decay of $P_{c}(4380)$ pentaquark in a molecular picture," Phys. Lett. B 782, 694 (2018) [arXiv:1802.01384 [hep-ph]].

[17] S. S. Agaev, K. Azizi and H. Sundu, "The structure, mixing angle, mass and couplings of the light scalar $f_{0}(500)$ and $f_{0}(980)$ mesons," Phys. Lett. B 781, 279 (2018) [arXiv:1711.11553 [hep-ph]].

[18] S. S. Agaev, K. Azizi and H. Sundu, "Application of the QCD light cone sum rule to tetraquarks: the strong vertices $X_{b} X_{b} \rho$ and $X_{c} X_{c} \rho$," Phys. Rev. D 93, no. 11, 114036 (2016) [arXiv:1605.02496 [hep-ph]].

[19] T. M. Aliev, A. Ozpineci, M. Savci and V. S. Zamiralov, "Vector meson-baryon strong coupling contants in light cone QCD sum rules," Phys. Rev. D 80, 016010 (2009) [arXiv:0905.4664 [hep-ph]]. 
[20] T. M. Aliev, K. Azizi and M. Savci, "Strong Coupling Constants of Decuplet Baryons with Vector Mesons," Phys. Rev. D 82, 096006 (2010) [arXiv:1007.3389 [hep-ph]].

[21] T. M. Aliev, K. Azizi and M. Savci, "Strong coupling constants of light pseudoscalar mesons with heavy baryons in QCD," Phys. Lett. B 696, 220 (2011) [arXiv:1009.3658 [hep-ph]].

[22] T. M. Aliev, K. Azizi and M. Savci, "Strong coupling constants of heavy spin-3/2 baryons with light pseudoscalar mesons," Nucl. Phys. A 870-871, 58 (2011) [arXiv:1102.5460 [hep$\mathrm{ph}]$.

[23] S. S. Agaev, K. Azizi and H. Sundu, "Decay widths of the excited $\Omega_{b}$ baryons," Phys. Rev. D 96, no. 9, 094011 (2017) [arXiv:1708.07348 [hep-ph]].

[24] W. Roberts and M. Pervin, "Heavy baryons in a quark model," Int. J. Mod. Phys. A 23, 2817 (2008) [arXiv:0711.2492 [nucl-th]].

[25] A. Valcarce, H. Garcilazo and J. Vijande, "Towards an understanding of heavy baryon spectroscopy," Eur. Phys. J. A 37, 217 (2008) [arXiv:0807.2973 [hep-ph]].

[26] S. S. Agaev, K. Azizi and H. Sundu, "Mass and decay constant of the newly observed exotic X(5568) state," Phys. Rev. D 93, no. 7, 074024 (2016) [arXiv:1602.08642 [hep-ph]].

[27] Q. F. L and Y. B. Dong, "Masses of open charm and bottom tetraquark states in a relativized quark model," Phys. Rev. D 94, no. 9, 094041 (2016) [arXiv:1603.06417 [hep$\mathrm{ph}]$.

[28] Z. G. Wang, "Analysis of the $\frac{1}{2}^{ \pm}$antitriplet heavy baryon states with QCD sum rules," Eur. Phys. J. C 68, 479 (2010) [arXiv:1001.1652 [hep-ph]].

[29] Z. G. Wang, "Reanalysis of the heavy baryon states Omega(b), Omega(c), Xi'(b), Xi'(c), Sigma(b) and Sigma(c) with QCD sum rules," Phys. Lett. B 685, 59 (2010) [arXiv:0912.1648 [hep-ph]].

[30] S. S. Agaev, K. Azizi and H. Sundu, "On the nature of the newly discovered $\Omega$ states," EPL 118, no. 6, 61001 (2017) [arXiv:1703.07091 [hep-ph]].

[31] D. Ebert, R. N. Faustov and V. O. Galkin, "Spectroscopy and Regge trajectories of heavy baryons in the relativistic quark-diquark picture," Phys. Rev. D 84, 014025 (2011) doi:10.1103/PhysRevD.84.014025 [arXiv:1105.0583 [hep-ph]].

[32] T. M. Aliev, K. Azizi and M. Savci, "Doubly Heavy Spin-1/2 Baryon Spectrum in QCD," Nucl. Phys. A 895, 59 (2012) [arXiv:1205.2873 [hep-ph]].

[33] T. M. Aliev, K. Azizi and M. Savc, "Mixing angle of doubly heavy baryons in QCD," Phys. Lett. B 715, 149 (2012) [arXiv:1205.6320 [hep-ph]].

[34] T. M. Aliev, K. Azizi and M. Savci, "The masses and residues of doubly heavy spin-3/2 baryons," J. Phys. G 40, 065003 (2013) [arXiv:1208.1976 [hep-ph]].

[35] T. M. Aliev, K. Azizi and M. Savci, "Masses and Residues of the Triply Heavy Spin-1/2 Baryons," JHEP 1304, 042 (2013) [arXiv:1212.6065 [hep-ph]].

[36] T. M. Aliev, K. Azizi and M. Savc, "Properties of triply heavy spin-3/2 baryons," J. Phys. G 41, 065003 (2014) [arXiv:1404.2091 [hep-ph]].

[37] M. N. Anwar, J. Ferretti, F. K. Guo, E. Santopinto and B. S. Zou, arXiv:1710.02540 [hep-ph]. 
[38] D. Ebert, R. N. Faustov, V. O. Galkin and A. P. Martynenko, "Mass spectra of doubly heavy baryons in the relativistic quark model," Phys. Rev. D 66, 014008 (2002) [hep$\mathrm{ph} / 0201217]$.

[39] K. W. Wei, B. Chen and X. H. Guo, "Masses of doubly and triply charmed baryons," Phys. Rev. D 92, no. 7, 076008 (2015) [arXiv:1503.05184 [hep-ph]].

[40] Y. J. Shi, W. Wang, Y. Xing and J. Xu, "Weak Decays of Doubly Heavy Baryons: Multi-body Decay Channels," arXiv:1712.03830 [hep-ph].

[41] C. Y. Wang, C. Meng, Y. Q. Ma and K. T. Chao, "NLO Effects for Doubly Heavy Baryon in QCD Sum Rules," arXiv:1708.04563 [hep-ph].

[42] Application of Light Cone QCD Sum Rules to Hadron Physics: Altuğ Özpineci, a PhD thesis submitted to the graduate school of natural and applied sciences, Middle East Technical University (2001).

[43] M. A. Shifman, A. I. Vainshtein and V. I. Zakharov, "QCD and Resonance Physics. Theoretical Foundations," Nucl. Phys. B 147, 385 (1979).

[44] I. I. Balitsky, V. M. Braun and A. V. Kolesnichenko, "Radiative Decay $\Sigma^{+} \rightarrow p \gamma$ in Quantum Chromodynamics," Nucl. Phys. B 312, 509 (1989).

[45] I. I. Balitsky and V. M. Braun, "Evolution Equations for QCD String Operators," Nucl. Phys. B 311, 541 (1989).

[46] L. J. Reinders, H. Rubinstein and S. Yazaki, "Hadron Properties from QCD Sum Rules," Phys. Rept. 127, 1 (1985).

[47] F. X. Lee and X. y. Liu, "Predictive ability of QCD sum rules for excited baryons," Phys. Rev. D 66, 014014 (2002) [nucl-th/0203051].

[48] U. Ozdem and K. Azizi, "Magnetic and quadrupole moments of the $Z_{c}(3900)$," Phys. Rev. D 96, no. 7, 074030 (2017) [arXiv:1707.09612 [hep-ph]].

[49] P. Ball, "Theoretical update of pseudoscalar meson distribution amplitudes of higher twist: The Nonsinglet case," JHEP 9901, 010 (1999), [hep-ph/9812375].

[50] P. Ball, V. M. Braun and A. Lenz, "Higher-twist distribution amplitudes of the K meson in QCD," JHEP 0605, 004 (2006) [hep-ph/0603063].

[51] P. Ball and R. Zwicky, "New results on $B \rightarrow \pi, K, \eta$ decay formfactors from light-cone sum rules," Phys. Rev. D 71, 014015 (2005) [hep-ph/0406232]. 